\title{
Valorization of Chemical Wastes: Ir(biscarbene)-Catalyzed Transfer Hydrogenation of Inorganic Carbonates Using Glycerol
}

\author{
Yeon-Joo Cheong, ${ }^{a}$ Kihyuk Sung, ${ }^{a}$ Sehoon Park, ${ }^{b}$ Jaehoon Jung, ${ }^{b, *}$ Hye-Young Jang ${ }^{a, *}$ \\ ${ }^{a}$ Department of Energy Systems Research, Ajou University, Suwon 16499, Korea \\ ${ }^{b}$ Department of Chemistry, University of Ulsan, Ulsan 44610, Korea
}

*Corresponding Author:

Email: hyjang2@ajou.ac.kr

Email: jjung2015@ulsan.ac.kr

Number of pages: 38

Number of Figures: 9

Number of Tables: 4

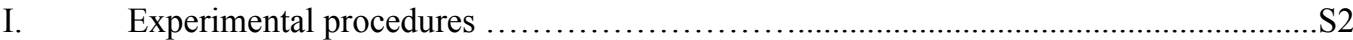

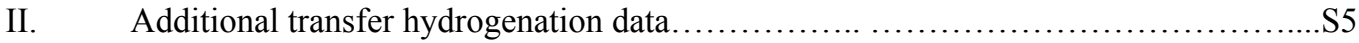

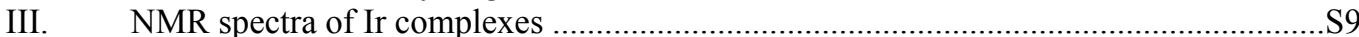

IV. Analysis of recovered Ir catalyst 1 from the reaction mixture ..........................S30

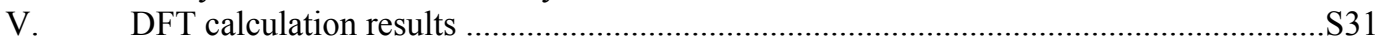




\section{Experimental procedure}

General Procedure. The $[\operatorname{Ir}(\mathrm{COD}) \mathrm{Cl}]_{2}, \mathbf{4}$, and 5 were used as received from Strem chemicals, Inc. 1, 1'Methylenebis(3-ethyl-1H-imidazole-3-ium) diiodide (LEt1[1- $\left.]_{2}\right), \quad$ 1,1'-(propane-1,3-diyl)bis(3-methyl-1Himidazol-3-ium) dibromide (LMe3[Br- $\left.]_{2}\right)$, and 1,1'-(propane-1,3-diyl)bis(3-ethyl-1 $H$-imidazol-3-ium) dibromide $\left(\mathrm{LEt} 3\left[\mathrm{Br}^{-}\right]_{2}\right)$ were prepared according to literature procedures. ${ }^{1,2}$ Proton nuclear magnetic resonance $\left({ }^{1} \mathrm{H} \mathrm{NMR}\right)$ spectra were recorded with a JEOL Resonance ECZ600R (600 MHz) spectrometer. Chemical shifts are reported in delta $(\delta)$ units, parts per million $(\mathrm{ppm})$ relative to the center of a singlet peak at $2.50 \mathrm{ppm}$ for DMSO- $\mathrm{d}_{6}$ or the singlet peak at $5.32 \mathrm{ppm}$ for $\mathrm{CD}_{2} \mathrm{Cl}_{2}$. Carbon-13 nuclear magnetic resonance $\left({ }^{13} \mathrm{C} \mathrm{NMR}\right)$ spectra were recorded with a JEOL Resonance ECZ600R (151 MHz). Chemical shift are reported in delta $(\delta)$ units, parts per million $(\mathrm{ppm})$ relative to the center of a multiplet peak at $39.52 \mathrm{ppm}$ for DMSO- $\mathrm{d}_{6}$ or the singlet peak at $53.84 \mathrm{ppm}$ for $\mathrm{CD}_{2} \mathrm{Cl}_{2}$. High resolution mass spectra were obtained with a magnetic sector-electric sector double focusing mass analyzer equipment. IR spectra were recorded with a FTIR spectrometer. Melting points of the compounds were checked in open capillary and were uncorrected. Elemental analyses were performed by Flash 2000 (Thermo Scienetific).

\section{Synthetic Procedure for the synthesis of 1 .}

Sodium hydride (44 mg, $1.1 \mathrm{mmol}$ ) was dissolved in $6 \mathrm{ml}$ of ethanol, which was added to a suspension of $[\operatorname{Ir}(\mathrm{COD}) \mathrm{Cl}]_{2}(134 \mathrm{mg}, 0.2 \mathrm{mmol})$ in ethanol $(6 \mathrm{ml})$. The reaction mixture was stirred for $1 \mathrm{~h}$ at room temperature, and 1,1'-(propane-1,3-diyl)bis(3-methyl-1H-imidazole-3-ium) dibromide (183 $\mathrm{mg}, 0.5 \mathrm{mmol})$ was added and stirred for $12 \mathrm{~h}$ at room temperature. The mixture solution was filtered with celite, and the filtrate was reduced in vacuo. The solid was dissolved with $\mathrm{CH}_{2} \mathrm{Cl}_{2}$ and the solution was filtered through the syringe filter. The filtrate was reduced in vacuo. Complex 1 was obtained as an orange solid in $98 \%$ yield.

\section{Synthetic Procedure for the synthesis of $\mathbf{1}^{\prime}$.}

Complex 1 (100 mg, $0.17 \mathrm{mmol})$ was dissolved in $5.7 \mathrm{ml}$ of $\mathrm{CH}_{2} \mathrm{Cl}_{2}$ under $\mathrm{CO}$ (1 atm) and stirred for $24 \mathrm{~h}$ at room temperature. Then, the solvent with volatile organic compounds was removed in vacuo. The yellow solid was washed with diethyl ether and complex 1' was obtained as a yellow solid in $91 \%$ yield.

\section{Synthetic Procedure for the synthesis of 1".}

Sodium hydride (35 mg, $0.875 \mathrm{mmol}$ ) was dissolved in ethanol $(4.4 \mathrm{ml})$, which was slowly added to a suspension of $\left[\operatorname{Ir}(\mathrm{COD}) \mathrm{Cl}_{2}(148 \mathrm{mg}, 0.22 \mathrm{mmol})\right.$ in $2.9 \mathrm{ml}$ of ethanol. The reaction mixture was stirred for $1 \mathrm{~h}$ at room temperature and 1,1'-(propane-1,3-diyl)bis(3-ethyl-1 $H$-imidazole-3-ium) dibromide (197 $\mathrm{mg}, 0.5 \mathrm{mmol}$ ) was added and stirred for $12 \mathrm{~h}$ at room temperature. Sodium iodide $(255 \mathrm{mg}, 1.7 \mathrm{mmol})$ was added to this mixture and stirred for another $12 \mathrm{~h}$ at room temperature. Then, the mixture was dried under reduced pressure and the product

\footnotetext{
${ }^{1}$ Meier, S. B.; Sarfert, W.; Junquera-Hernandez, J. M.; Delgado, M.; Tordera, D.; Orti, E.; Bolink, H. J.; Kessler, F.; Scopelliti, R.; Gratzel, M.; Nazeeruddin M. K. and Baranoff, E. A deep-blue emitting charged bis-cyclometallated iridium(III) complex for light-emitting electrochemical cells. J. Mater. Chem. C 2013, 1, 58-68.

${ }^{2}$ Nachtigall, F. M.; Corilo, Y. E.; Cassol, C. C.; Ebeling, G.; Morgon, N. H.; Dupont J. and Eberlin, M. N. Multiply charged (di-)radicals. Angew. Chem. Int. Ed. 2008, 47, 151-154.
} 
was purified by column chromatography $\left(\mathrm{CH}_{2} \mathrm{Cl}_{2} / \mathrm{Acetone}=8: 2\right)$. Complex $\mathbf{1}^{\prime \prime}$ was obtained as an orange solid in $72 \%$ yield.

\section{Synthetic Procedure for the synthesis of 2.}

Sodium hydride $(35 \mathrm{mg}, 0.875 \mathrm{mmol})$ was dissolved in ethanol $(4.4 \mathrm{ml})$ and slowly added to a suspension of $[\operatorname{Ir}(\mathrm{COD}) \mathrm{Cl}]_{2}(148 \mathrm{mg}, 0.22 \mathrm{mmol})$ in ethanol $(2.9 \mathrm{ml})$. The reaction mixture was stirred for $1 \mathrm{~h}$ at room temperature and 1,1'-(propane-1,3-diyl)bis(3-methyl-1H-imidazole-3-ium) dibromide (183 mg, $0.5 \mathrm{mmol}$ ) was added and stirred during $12 \mathrm{~h}$ at room temperature. Sodium iodide $(255 \mathrm{mg}, 1.7 \mathrm{mmol})$ was added and stirred for another $12 \mathrm{~h}$ at room temperature. Then, the mixture was dried under reduced pressure, and the product was purified by column chromatography $\left(\mathrm{CH}_{2} \mathrm{Cl}_{2} / \mathrm{Acetone}=8: 2\right)$. Complex 2 was obtained as an orange solid in $72 \%$ yield.

\section{Synthetic Procedure for $2^{\prime}$.}

Complex $2(100 \mathrm{mg}, 0.16 \mathrm{mmol})$ was dissolved in $5.3 \mathrm{ml} \mathrm{CH}_{2} \mathrm{Cl}_{2}$ of under $\mathrm{CO}(1 \mathrm{~atm})$ and stirred for $24 \mathrm{~h}$ at room temperature. Then, the volatiles were removed in vacuo. The yellow solid was washed with diethyl ether and complex $\mathbf{2}^{\prime}$ was obtained as a yellow solid in $66 \%$ yield.

\section{Synthetic Procedure for 3.}

Complex $2(100 \mathrm{mg}, 0.16 \mathrm{mmol})$ and $\mathrm{AgPF}_{6}(40 \mathrm{mg}, 0.16 \mathrm{mmol})$ was stirred in $8 \mathrm{ml}$ of $\mathrm{MeCN}$ for $1 \mathrm{~h}$ at room temperature without light. Then, the mixture was filtered through celite to remove AgX. The filtrate was dried under reduced pressure. Complex $\mathbf{3}$ was obtained as an orange solid in $55 \%$ yield.

\section{Synthetic Procedure for 6 .}

Potassium tert-butoxide ( $40 \mathrm{mg}, 0.35 \mathrm{mmol}$ ) was dissolved in $32 \mathrm{ml}$ of THF, which was added slowly to a stirred suspension of 1,1'-methylenebis(3-ethyl-1H-imidazole-3-ium) diiodide (101 $\mathrm{mg}, 0.22 \mathrm{mmol}$ ) in THF (30 ml). The reaction mixture was stirred for $1 \mathrm{~h}$ at room temperature. $[\operatorname{Ir}(\mathrm{COD}) \mathrm{Cl}]_{2}(67 \mathrm{mg}, 0.1 \mathrm{mmol})$ in toluene $(20 \mathrm{ml})$ was added to the above-mixture and stirred for $12 \mathrm{~h}$ at $80^{\circ} \mathrm{C}$. Then, the reactant was cooled down to ambient temperature. To this mixture, $0.2 \mathrm{mmol}$ of $\mathrm{KPF}_{6}$ was added and stirred for another $1 \mathrm{~h}$. Solid compounds were removed by celite filtration, and the filtrate was purified by column chromatography with $\mathrm{CH}_{2} \mathrm{Cl}_{2}$. For the further purification, complex 6 was obtained as a yellow solid in $12 \%$ yield after recrystallization with diethyl ether/hexane.

\section{Catalytic procedure for the transfer hydrogenation of $\mathrm{K}_{2} \mathrm{CO}_{3}$ in glycerol}

The catalyst $(0.14 \mu \mathrm{mol}), \mathrm{K}_{2} \mathrm{CO}_{3}(4 \mathrm{mmol})$, EtOH $(0.05 \mathrm{mmol})$ and glycerol $(1 \mathrm{ml})$ were put into a seal tube. The mixture was heated at $150{ }^{\circ} \mathrm{C}$ or $180{ }^{\circ} \mathrm{C}$ for $20 \mathrm{~h}$. Then, the reaction mixture was cooled down to ambient temperature, and diluted with $\mathrm{H}_{2} \mathrm{O}$. The TONs of formats and lactates were calculated by ${ }^{1} \mathrm{H}$ NMR spectroscopy in $\mathrm{D}_{2} \mathrm{O}$, using isonicotinic acid as an internal standard. 


\section{Determination of TONs of formate and lactate by ${ }^{1} \mathrm{H}$ NMR}

After reaction, the reaction mixtures were diluted with $9 \mathrm{ml} \mathrm{H}_{2} \mathrm{O}$. Isonicotinic acid $(0.05 \mathrm{mmol})$ as an internal standard was added to the diluted solution. The $0.45 \mathrm{ml}$ of the diluted solution and $0.15 \mathrm{ml}$ of $\mathrm{D}_{2} \mathrm{O}$ were mixed for NMR analysis. The moles of the products were calculated by integration values of the signature peak of each species based on moles of the internal standard.

\section{Conversion of glycerol to lactate determined by ${ }^{1} \mathrm{H}$ NMR}

After reaction, the reaction mixtures were diluted with $9 \mathrm{ml} \mathrm{H}_{2} \mathrm{O}$. Isonicotinic acid $(0.05 \mathrm{mmol})$ as an internal standard was added to the diluted solution. The $0.45 \mathrm{ml}$ of the diluted solution and $0.15 \mathrm{ml}$ of $\mathrm{D}_{2} \mathrm{O}$ were mixed for NMR analysis. The moles of lactate were calculated by integration values of the signature peak of each species based on moles of the internal standard. The only by-products are propylene glycol. In the ${ }^{1} \mathrm{H}$ NMR spectrum of the mixture, formate, lactate, propylene glycol, ethanol (used as a reagent), and remaining glycerol were detected. As an example, ${ }^{1} \mathrm{H}$ NMR spectrum of entry 3 (Table 1 ) is shown below.

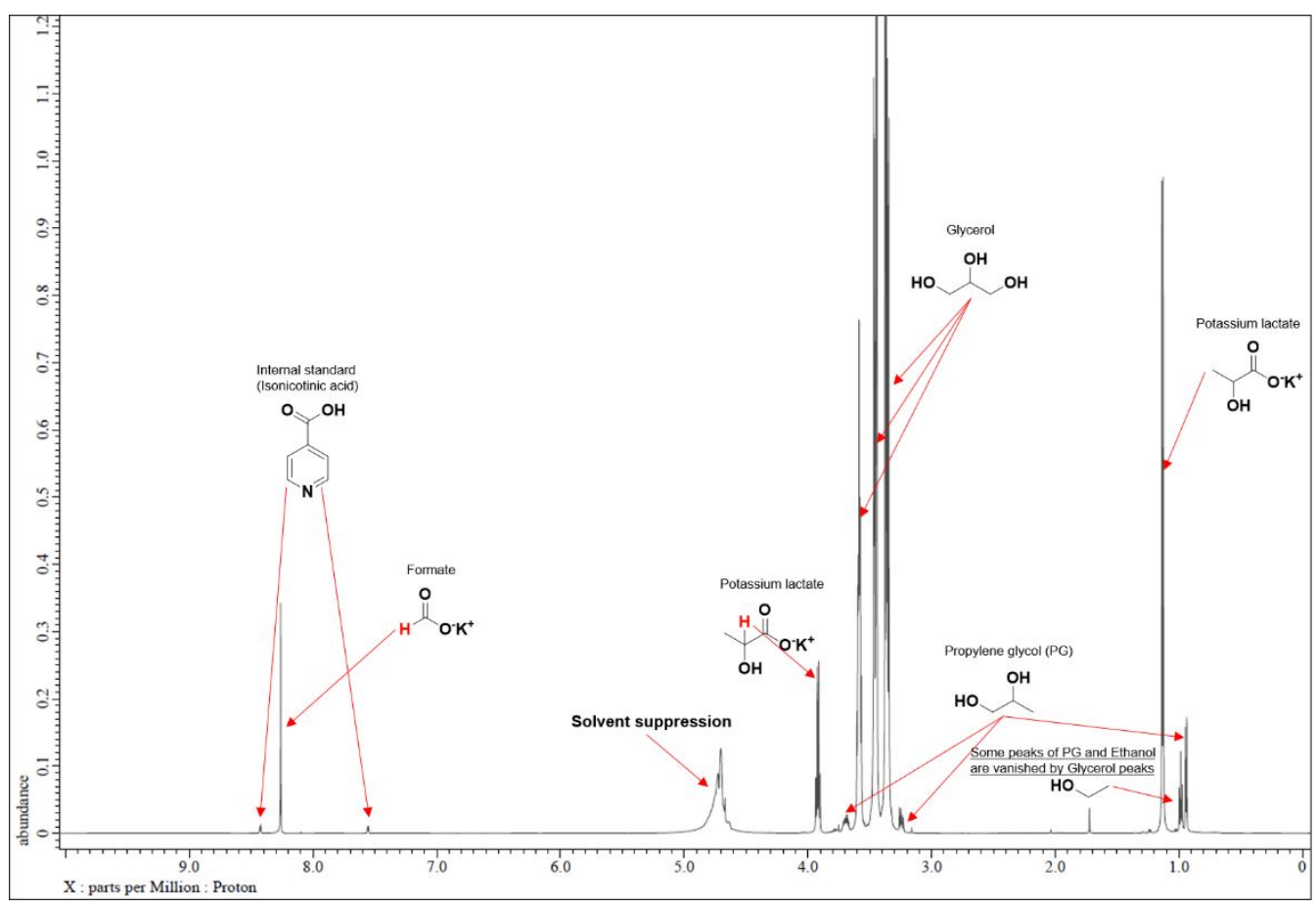

Figure S1. ${ }^{1} \mathrm{H}$ NMR spectrum of the reaction mixture of entry 3 (Table 1) 


\section{Additional transfer hydrogenation data}

Table S1. Transfer hydrogenation varying the amount of $\mathrm{K}_{2} \mathrm{CO}_{3}$ and additives

\begin{tabular}{ccccc} 
entry & $\mathrm{K}_{2} \mathrm{CO}_{3}$ & additive & formate (TON) & lactate (TON) \\
\hline 1 & $2 \mathrm{mmol}$ & $\mathrm{EtOH}(0.05 \mathrm{ml})$ & 4964 & 11206 \\
2 & $2 \mathrm{mmol}$ & $\mathrm{EtOH}(0.1 \mathrm{ml})$ & 3300 & 8282 \\
3 & $2 \mathrm{mmol}$ & $\mathrm{H}_{2} \mathrm{O}(0.1 \mathrm{ml})$ & 4000 & 11359 \\
4 & $4 \mathrm{mmol}$ & $\mathrm{EtOH}(0.05 \mathrm{ml})$ & 10764 & 22245
\end{tabular}

The mixture of catalyst $1^{\prime}(0.14 \mu \mathrm{mol}), \mathrm{K}_{2} \mathrm{CO}_{3}$, and additives in glycerol $(1 \mathrm{ml})$ was heated at $150{ }^{\circ} \mathrm{C}$ for $20 \mathrm{~h}$.

Table S2. Catalyst recycling: accumulated TONs of formates and lactates

\begin{tabular}{cccc} 
entry & recycle & formate (TON) & lactate (TON) \\
\hline 1 & $1^{\text {st }}$ & 22812 & 67786 \\
2 & $2^{\text {nd }}$ & 30519 & 73396 \\
3 & $3^{\text {rd }}$ & 36901 & 89177
\end{tabular}

Procedure of recycling experiments

$1^{\text {st }}$ recycling: After the reaction including catalyst 1

$(0.14 \mu \mathrm{mol})$ in $\mathrm{EtOH}(0.05 \mathrm{ml}), \mathrm{K}_{2} \mathrm{CO}_{3}(4 \mathrm{mmol})$, and

glycerol (1 ml) was kept for $20 \mathrm{~h}, \mathrm{~K}_{2} \mathrm{CO}_{3}(4 \mathrm{mmol})$ and

glycerol $(1 \mathrm{ml})$ were added. The resulting TONs are

showns in entry 1.

$2^{\text {nd }}$ recycling: After the $1^{\text {st }}$ recycling experiment, $\mathrm{K}_{2} \mathrm{CO}_{3}$

$(4 \mathrm{mmol})$ and glycerol $(1 \mathrm{ml})$ were added. The resulting

TONs are showns in entry 2.

$3^{\text {rd }}$ recycling: After the $2^{\text {nd }}$ recycling experiment, $\mathrm{K}_{2} \mathrm{CO}_{3}$

$(4 \mathrm{mmol})$ and glycerol $(1 \mathrm{ml})$ were added. The resulting

TONs are showns in entry 3.

Table S3. Reactions in water solvent

\begin{tabular}{cccc} 
entry & temp & formate (TON) & lactate (TON) \\
\hline 1 & $150{ }^{\circ} \mathrm{C}$ & 728 & 6550 \\
2 & $180^{\circ} \mathrm{C}$ & 5621 & 29457
\end{tabular}

The mixture of catalyst $1(0.15 \mu \mathrm{mol}), \mathrm{K}_{2} \mathrm{CO}_{3}(4 \mathrm{mmol})$, and glycerol $(4 \mathrm{ml})$ in $\mathrm{H}_{2} \mathrm{O}(4 \mathrm{ml})$ was heated for $20 \mathrm{~h}$.

*entry 1: the reaction conditions reported by Choudhury in ACS Catal 2019 
Table S4. The effect of $\mathrm{KOH}$ in the transfer hydrogenation

\begin{tabular}{|c|c|c|c|c|}
\hline entry & carbonates & $\mathrm{KOH}$ & formate (TON) & lactate \\
\hline 1 & $\mathrm{KHCO}_{3}$ & $1 \mathrm{mmol}$ & 11143 & 14127 \\
\hline 2 & $\mathrm{KHCO}_{3}$ & $2 \mathrm{mmol}$ & 13089 & 16967 \\
\hline 3 & $\mathrm{KHCO}_{3}$ & $3 \mathrm{mmol}$ & 8494 & 17904 \\
\hline 4 & $\mathrm{KHCO}_{3}$ & $4 \mathrm{mmol}$ & 8975 & 26207 \\
\hline 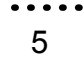 & $\mathrm{K}_{2} \mathrm{CO}_{3}$ & - & 16856 & 32609 \\
\hline 6 & $\mathrm{~K}_{2} \mathrm{CO}_{3}$ & $1 \mathrm{mmol}$ & 13403 & 34238 \\
\hline
\end{tabular}

The mixture of catalyst $1(0.14 \mu \mathrm{mol})$, carbonates $(4 \mathrm{mmol})$, $\mathrm{KOH}$, and $\mathrm{EtOH}(0.05 \mathrm{ml})$ in glycerol $(1 \mathrm{ml})$ was heated at $180{ }^{\circ} \mathrm{C}$ for $20 \mathrm{~h}$. 


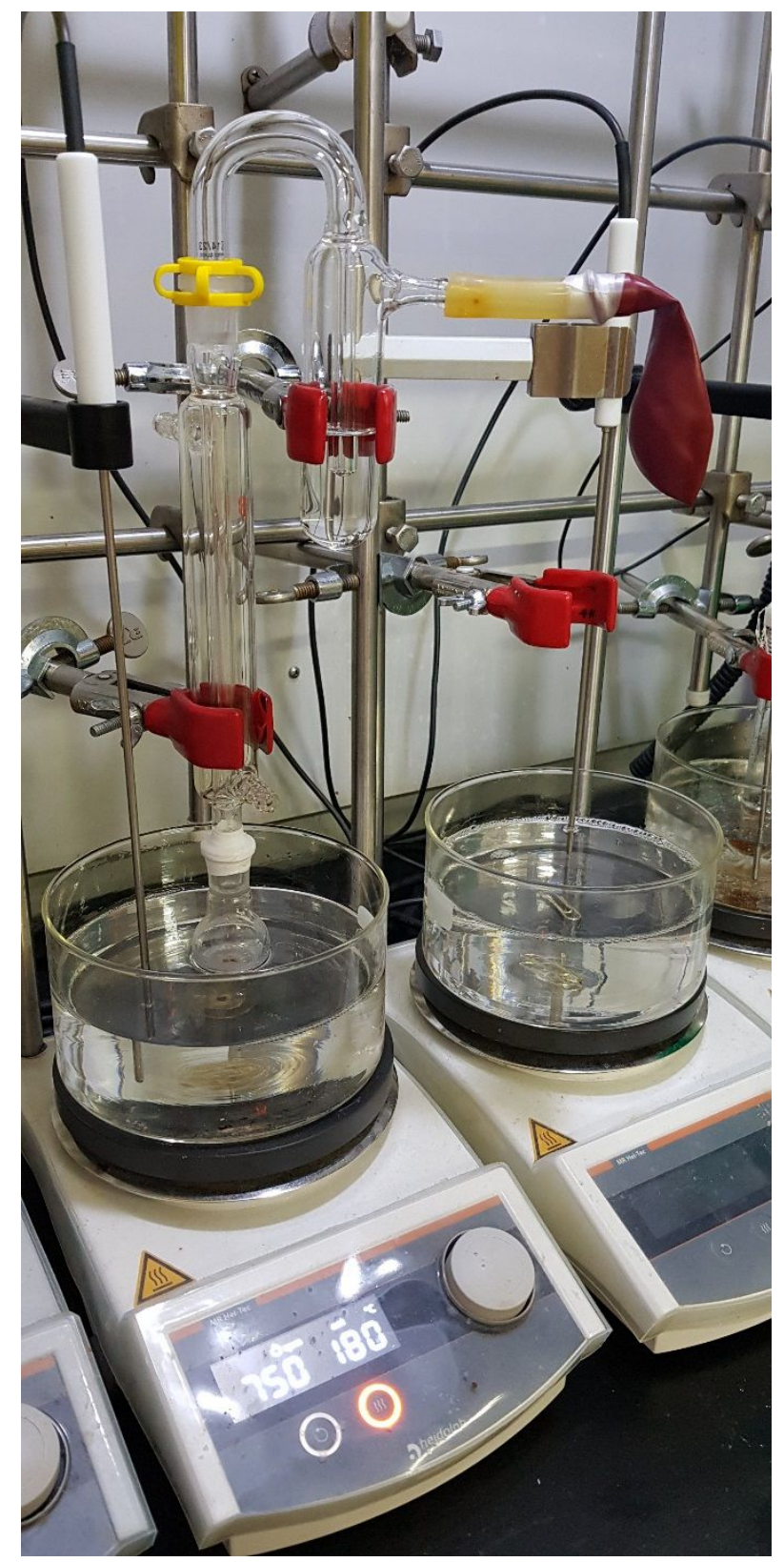

Figure S2. The experimental setting to check the $\mathrm{CO}_{2}$ generation from the reaction mixture

The catalyst $(0.14 \mu \mathrm{mol}), \mathrm{K}_{2} \mathrm{CO}_{3}(4 \mathrm{mmol}), \mathrm{EtOH}(0.05 \mathrm{mmol})$ and glycerol $(1 \mathrm{ml})$ were put into a $10 \mathrm{ml}$ round bottom flask which was connected with a bubbler. The bubbler was filled with $1 \mathrm{M} \mathrm{KOH}$ (or $\mathrm{NaOH}$ ) solution. The mixture was heated at $180{ }^{\circ} \mathrm{C}$ for $20 \mathrm{~h}$. The solution of $1 \mathrm{M} \mathrm{KOH}$ solution $(0.45 \mathrm{ml})$ was mixed with $0.15 \mathrm{ml} \mathrm{D}_{2} \mathrm{O}$ for NMR analysis.

${ }^{13} \mathrm{C}$ NMR spectrum

- $\quad$ The peak at 168 ppm corresponds to the carbonyl group of carbonates. The generated $\mathrm{CO}_{2}$ was trapped in $\mathrm{KOH}$ solution to form $\mathrm{KHCO}_{3}$. The peaks at 57.5 and $17.0 \mathrm{ppm}$ were assigned as ethanol. 

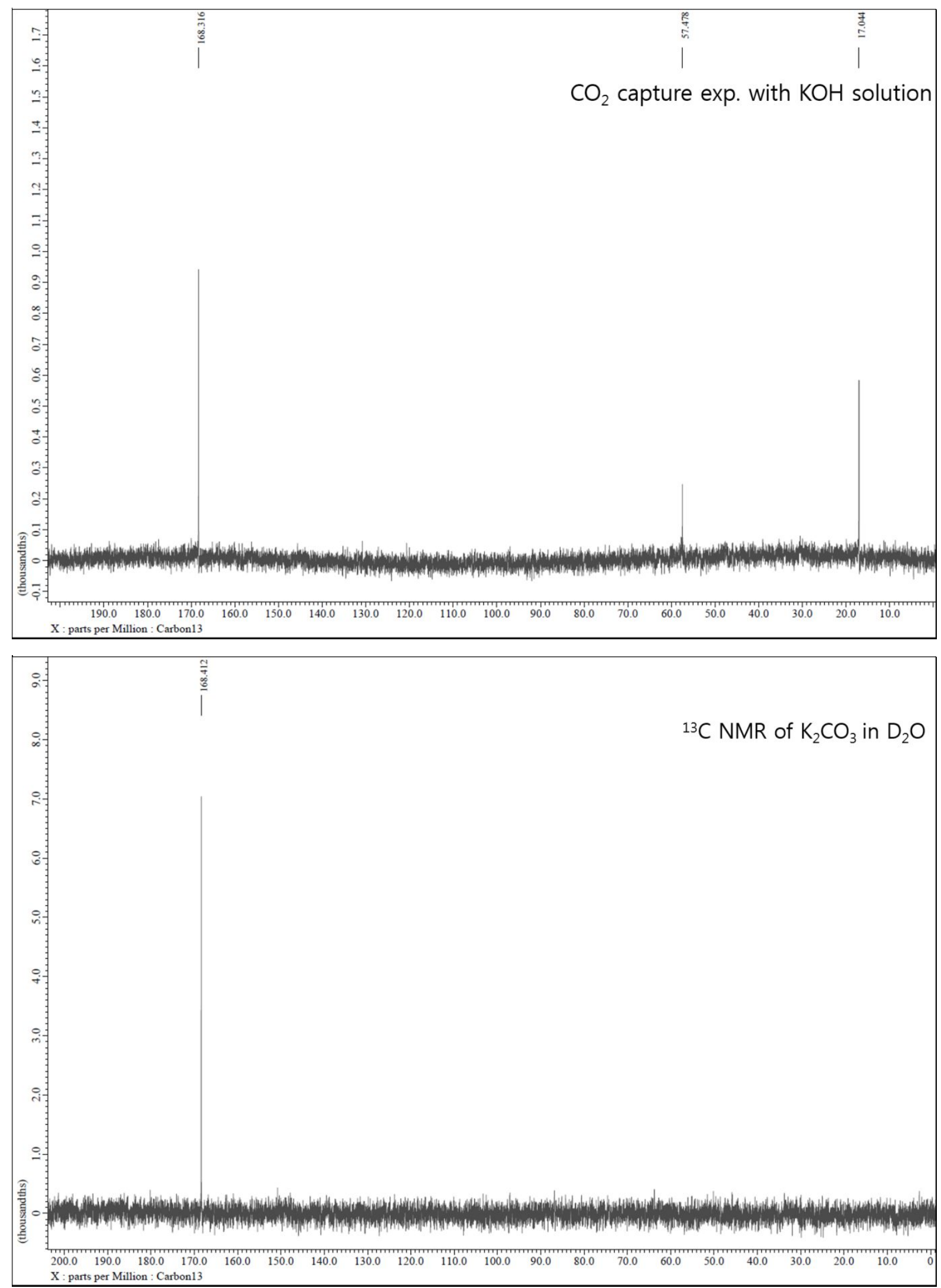


\section{NMR spectra of Ir complexes}

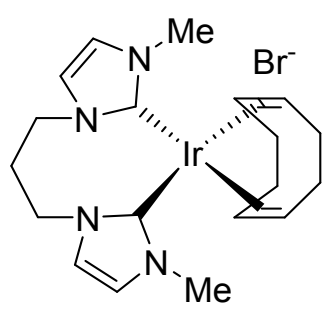

$\operatorname{Ir}(\mathrm{LMe3})(\mathrm{COD}) \mathrm{Br}(1)$ :

${ }^{1}$ H NMR (DMSO-d $\left.6,600 \mathrm{MHz}\right) \delta 7.28(\mathrm{~d}, J=1.4 \mathrm{~Hz}, 2 \mathrm{H}), 7.22(\mathrm{~d}, J=2.1 \mathrm{~Hz}, 2 \mathrm{H}), 4.59$ (dd, $J=14.5,11.0 \mathrm{~Hz}$, 2H), 4.30 (dd, $J=14.4,6.0 \mathrm{~Hz}, 2 \mathrm{H}), 3.98-4.08$ (m, 4H), 3.80 (s, 6H), 2.24-2.35 (m, 5H), 1.89-1.99 (m, 4H), 1.65$1.71(\mathrm{~m}, 1 \mathrm{H})$.

${ }^{13}$ C NMR (DMSO-d 6 , $\left.151 \mathrm{MHz}\right) \delta 176.75,122.38,122.27,75.49,74.24,51.38,36.99,33.13,30.80,30.77$.

HRMS $m / z$ (FAB, [M-Br] $]^{+}$: calculated for $\mathrm{C}_{19} \mathrm{H}_{28} \mathrm{~N}_{4} \mathrm{Ir}$ 505.1944, found: 505.1946 .

EA calculated for $\mathrm{C}_{19} \mathrm{H}_{28} \mathrm{BrIrN}_{4}$ (wt 584.58): C, 39.04; H, 4.83; N, 9.58, found: C, 39.09; H, 4.95; N, $9.56 \%$. Mp decomposed at $245{ }^{\circ} \mathrm{C}$. 


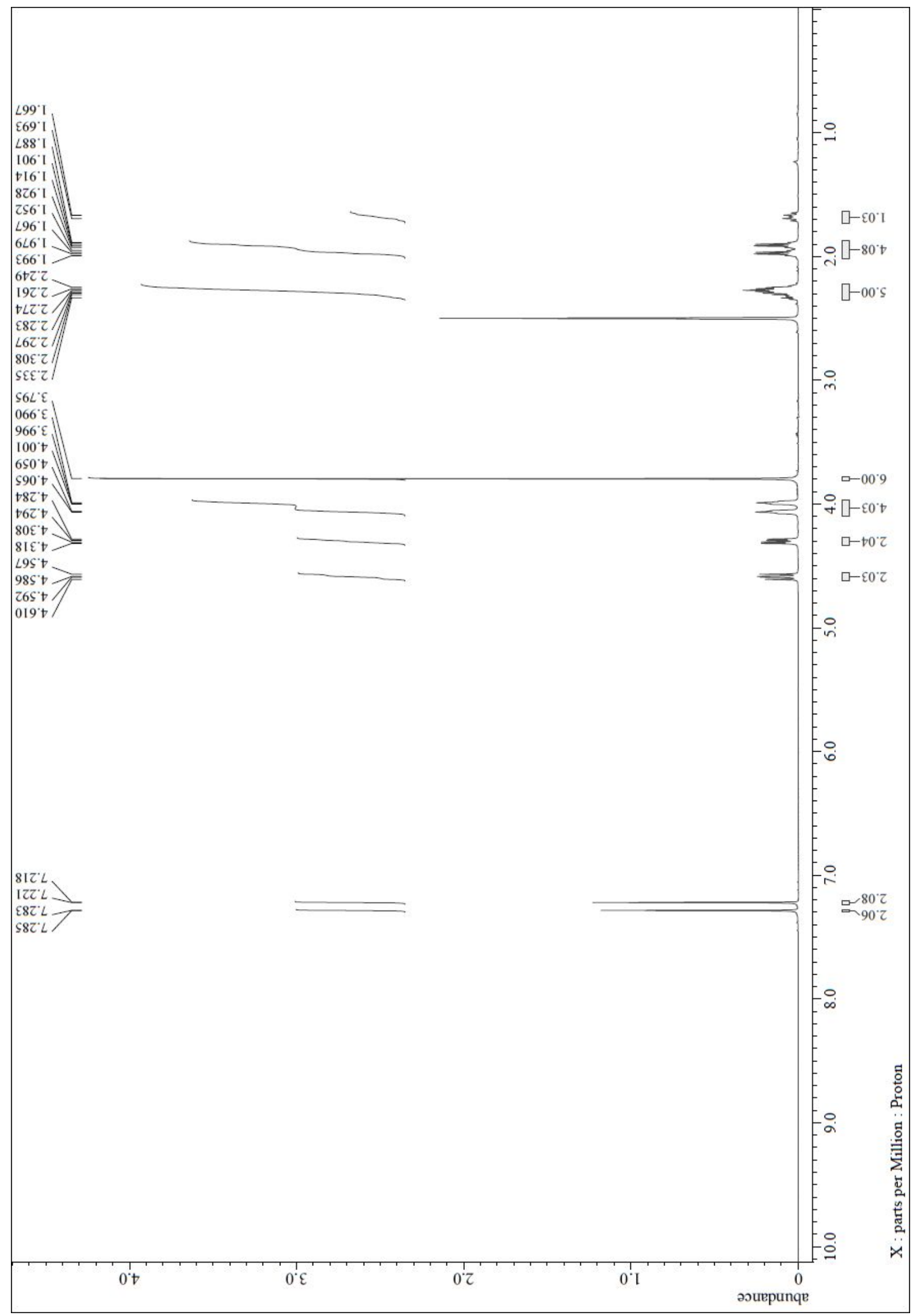




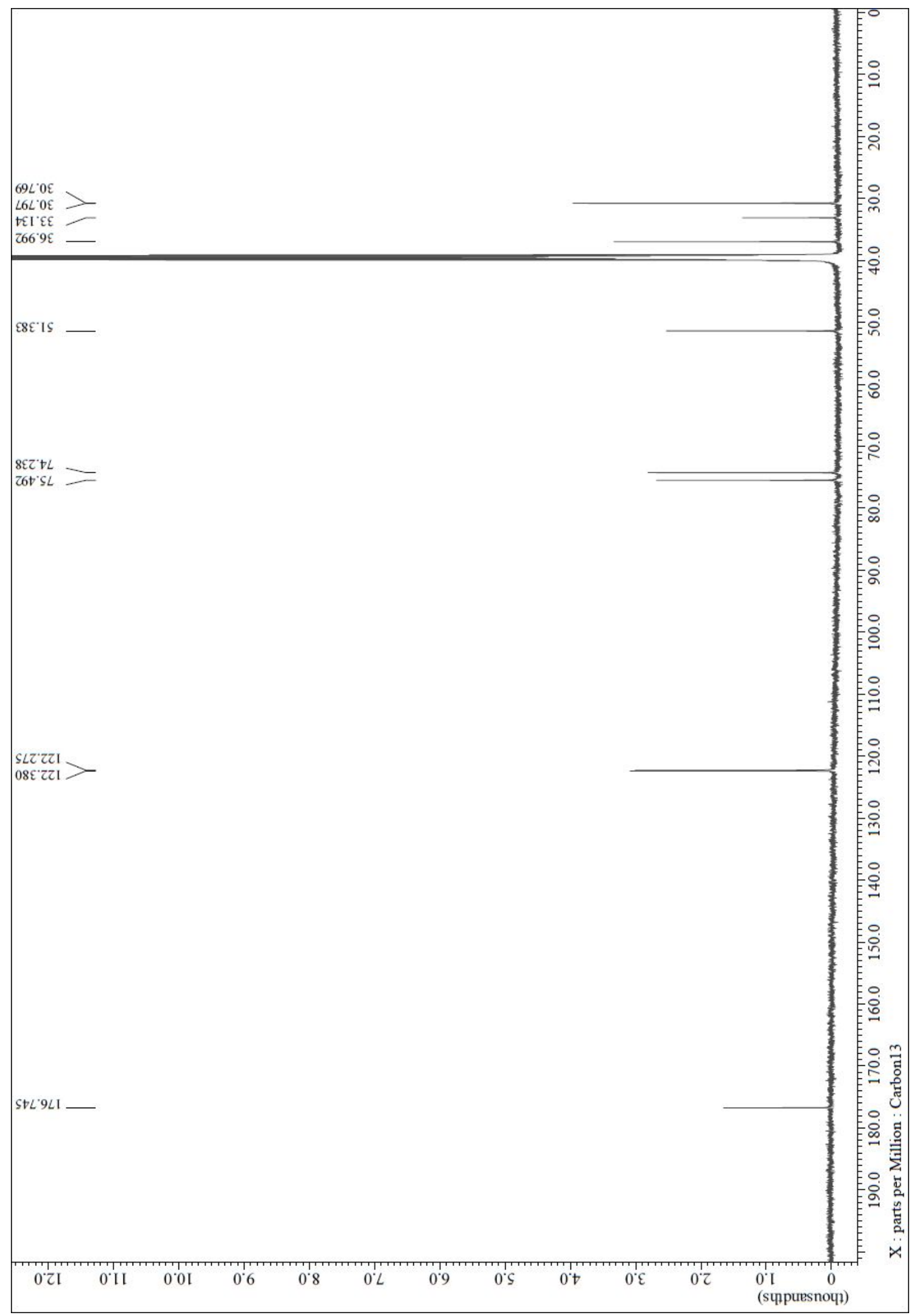




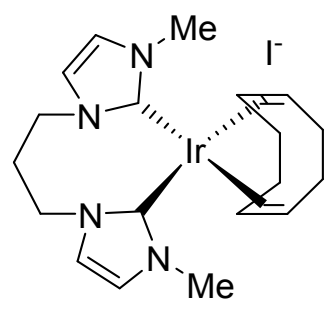

$\operatorname{Ir}(\mathrm{LMe3})(\mathrm{COD}) \mathrm{I}(2)$ :

${ }^{1}$ H NMR (DMSO-d 6 , $\left.600 \mathrm{MHz}\right) \delta 7.28(\mathrm{~d}, J=2.1 \mathrm{~Hz}, 2 \mathrm{H}), 7.22(\mathrm{~d}, J=2.1 \mathrm{~Hz}, 2 \mathrm{H}), 4.59(\mathrm{dd}, J=14.5,11.0 \mathrm{~Hz}$, 2H), 4.30 (dd, $J=14.4,6.0 \mathrm{~Hz}, 2 \mathrm{H}), 3.98-4.08$ (m, 4H), 3.79 (s, 6H), 2.23-2.35 (m, 5H), 1.89-1.99 (m, 4H), $1.65-$ $1.71(\mathrm{~m}, 1 \mathrm{H})$.

${ }^{13}$ C NMR (DMSO-d 6 , $\left.151 \mathrm{MHz}\right) \delta 176.76,122.38,122.28,75.50,74.26,51.39,37.00,33.13,30.82,30.78$.

HRMS $m / z$ (FAB, [M-I] $]^{+}$: calculated for $\mathrm{C}_{19} \mathrm{H}_{28} \mathrm{~N}_{4} \mathrm{Ir} 505.1944$, found: 505.1942.

EA calculated for $\mathrm{C}_{19} \mathrm{H}_{28} \mathrm{IIrN}{ }_{4}$ (wt 631.58): C, 36.13; H, 4.47; N, 8.87, found: C, 35.61; H, 4.49; N, 8.43 \%.

Mp decomposed at $240{ }^{\circ} \mathrm{C}$. 


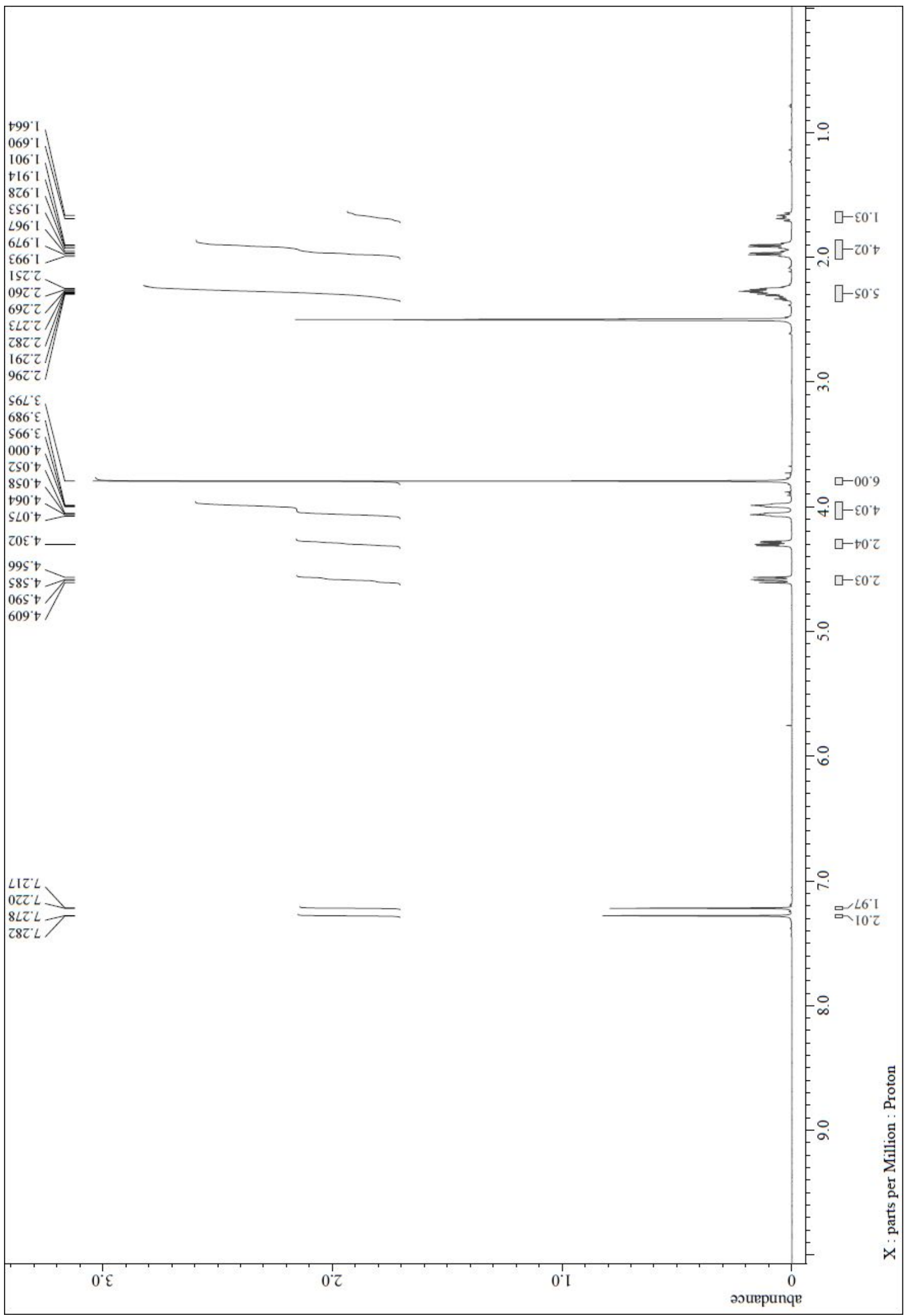




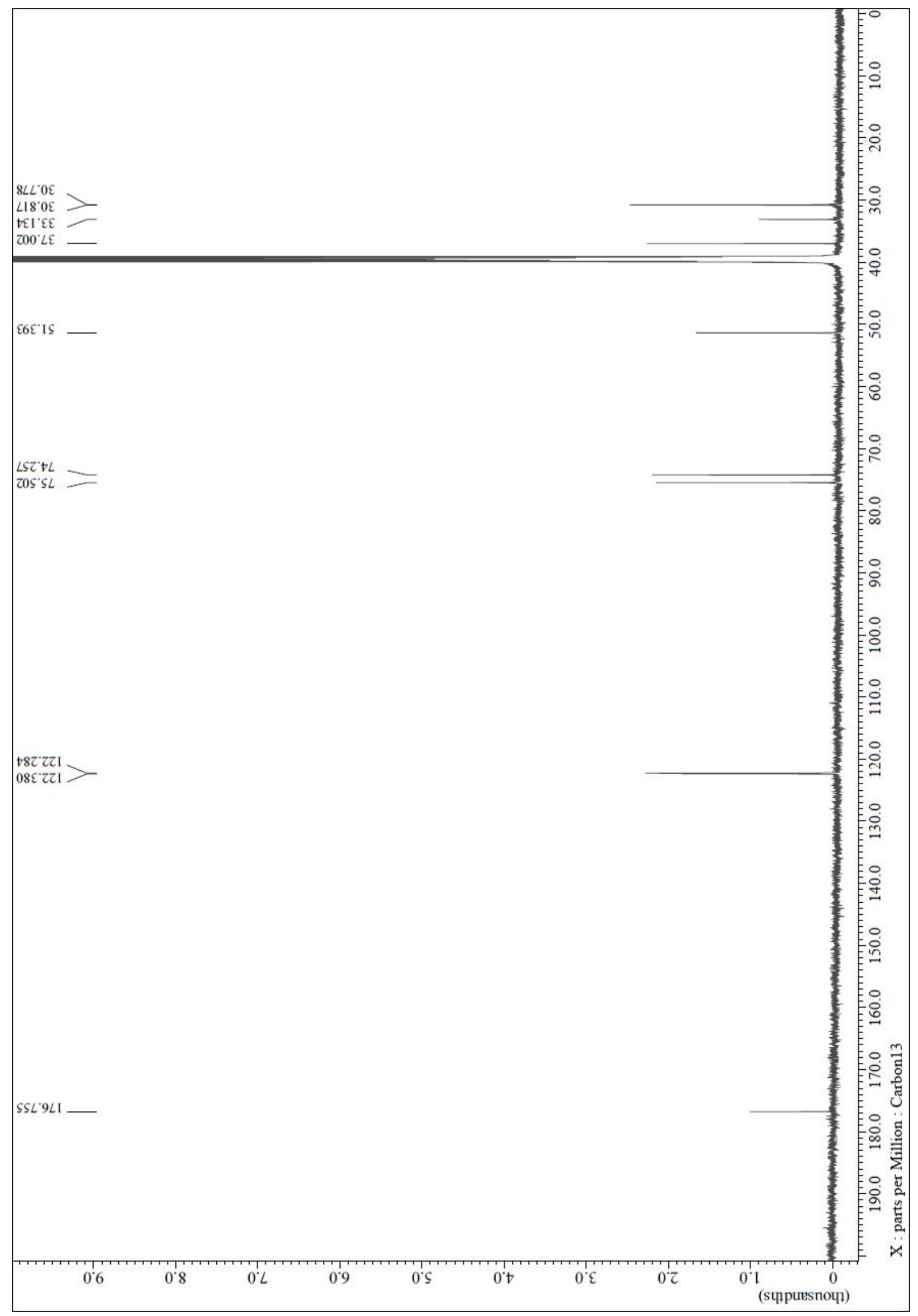




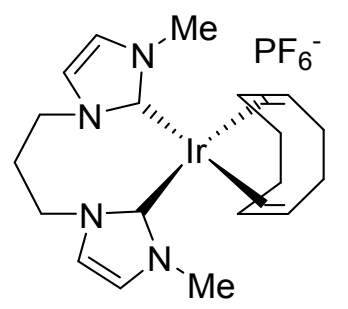

$\operatorname{Ir}\left(\mathrm{LMe}_{3}\right)(\mathrm{COD}) \mathrm{PF}_{6}(3)$ :

${ }^{1}$ H NMR (DMSO-d 6 , $\left.600 \mathrm{MHz}\right) \delta 7.27(\mathrm{~d}, J=1.4 \mathrm{~Hz}, 2 \mathrm{H}), 7.21(\mathrm{~d}, J=2.1 \mathrm{~Hz}, 2 \mathrm{H}), 4.59(\mathrm{dd}, J=14.5,11.0 \mathrm{~Hz}$, 2H), 4.29 (dd, $J=13.8,6.0 \mathrm{~Hz}, 2 \mathrm{H}), 3.98-4.08(\mathrm{~m}, 4 \mathrm{H}), 3.80(\mathrm{~s}, 6 \mathrm{H}), 2.24-2.35(\mathrm{~m}, 5 \mathrm{H}), 1.89-1.99(\mathrm{~m}, 4 \mathrm{H}), 1.65-$ $1.71(\mathrm{~m}, 1 \mathrm{H})$.

${ }^{13}$ C NMR (DMSO-d $\left.6,151 \mathrm{MHz}\right) \delta 176.76,122.37,122.27,75.51,74.27,51.39,36.98,33.12,30.81,30.77$.

HRMS $m / z\left(\mathrm{FAB},\left[\mathrm{M}-\mathrm{PF}_{6}\right]^{+}\right.$: calculated for $\mathrm{C}_{19} \mathrm{H}_{28} \mathrm{~N}_{4} \mathrm{Ir} 505.1944$, found: 505.1945 .

EA calculated for $\mathrm{C}_{19} \mathrm{H}_{28} \mathrm{~F}_{6} \mathrm{IrN}_{4} \mathrm{P}$ (wt 649.64): C, 35.13; H, 4.35; N, 8.62, found: C, 35.03; H, 4.24; N, 8.79 \%. Mp $250{ }^{\circ} \mathrm{C}$. 


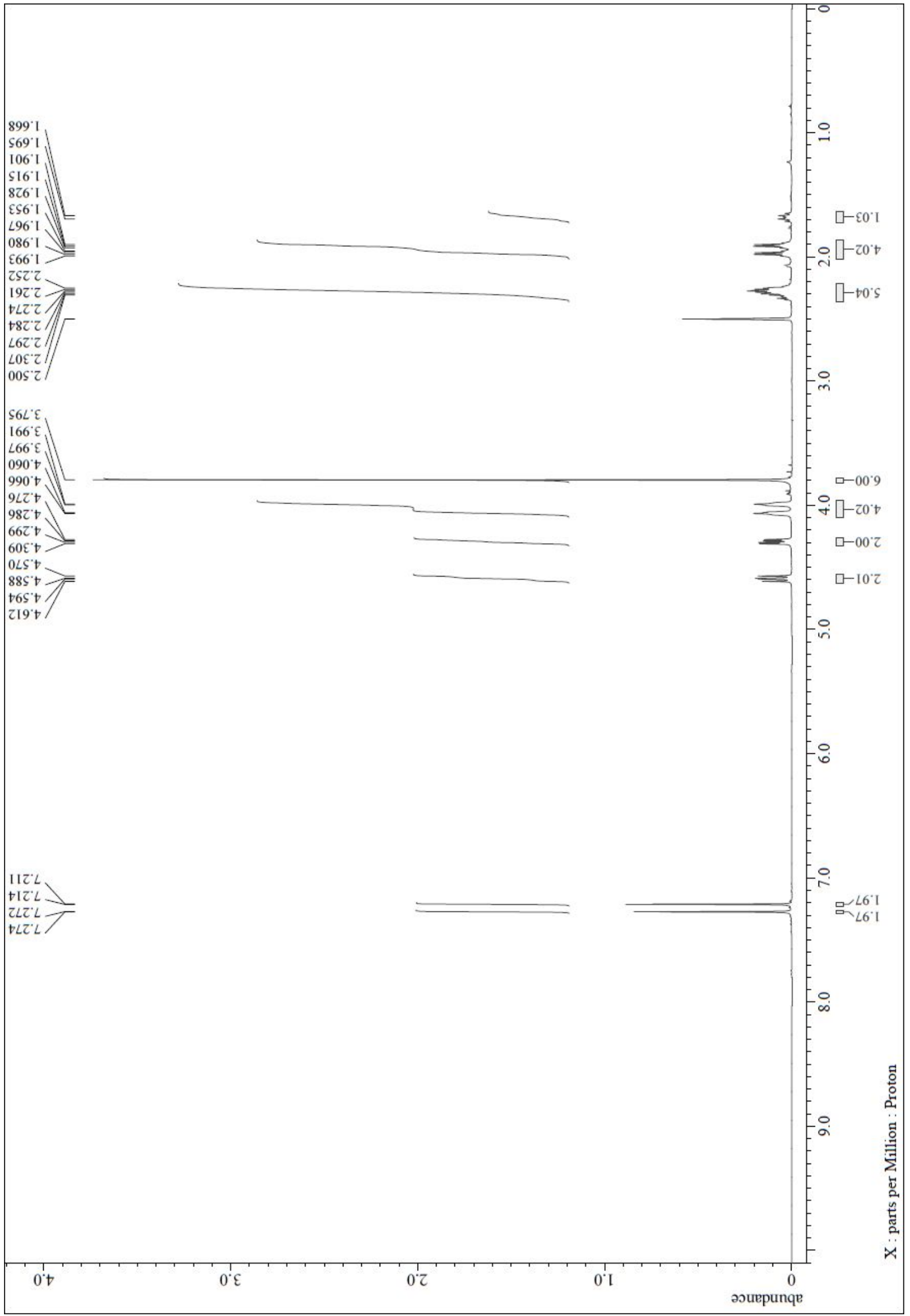




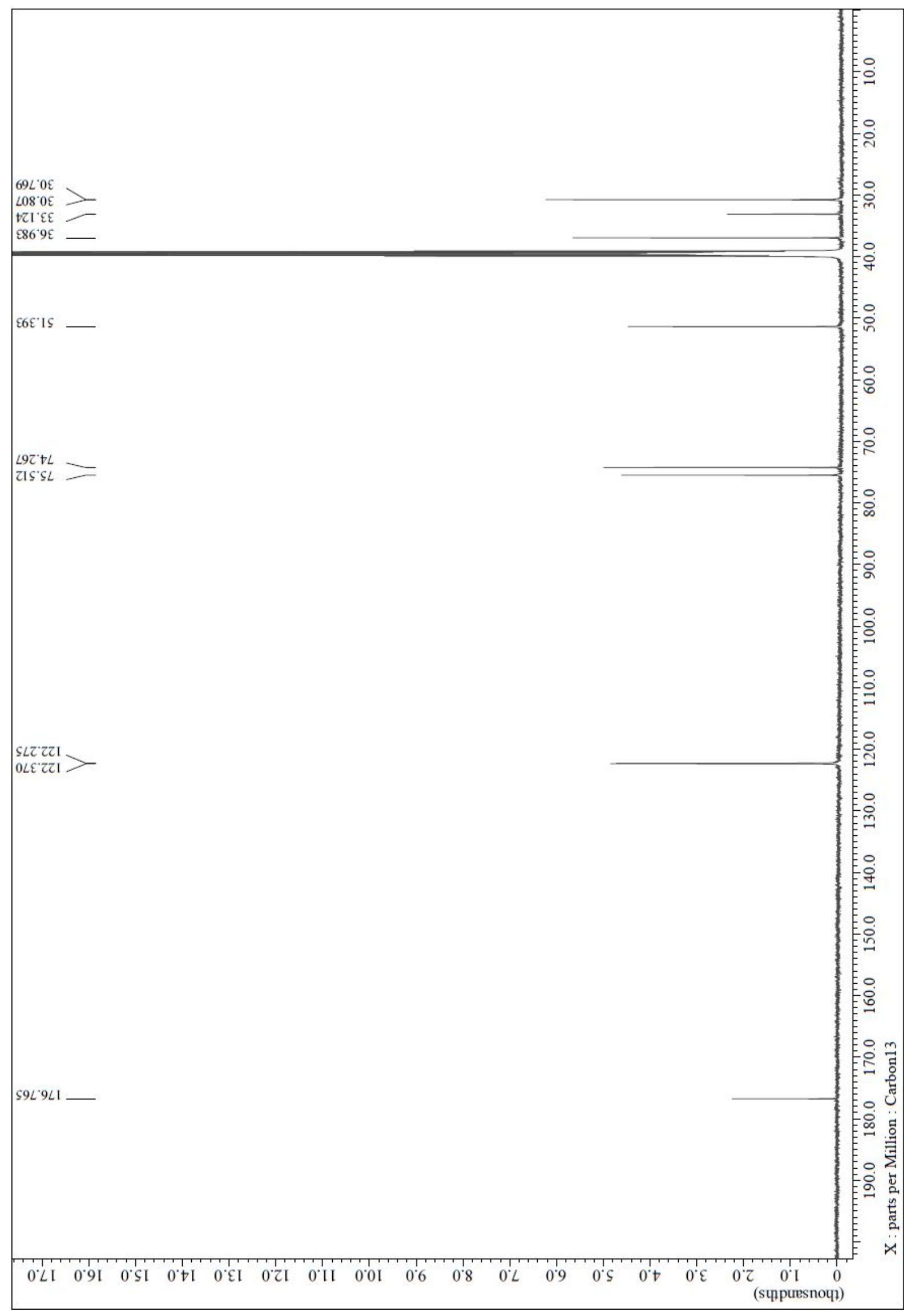




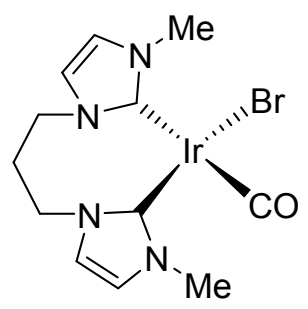

$\operatorname{Ir}(\mathrm{LMe} 3)(\mathrm{CO}) \mathrm{Br}\left(1^{\prime}\right)$ :

${ }^{1}$ H NMR (DMSO-d $\left.6,600 \mathrm{MHz}\right) \delta 7.47(\mathrm{~d}, J=2.1 \mathrm{~Hz}, 2 \mathrm{H}), 7.43(\mathrm{~d}, J=2.1 \mathrm{~Hz}, 2 \mathrm{H}), 4.53(\mathrm{dd}, J=14.5,11.0 \mathrm{~Hz}$, $2 \mathrm{H}), 4.35$ (dd, $J=14.5,5.5 \mathrm{~Hz}, 2 \mathrm{H}), 3.78$ (s, 6H), 2.24-2.30 (m, 1H), 1.78-1.84 (m, $1 \mathrm{H})$.

${ }^{13}$ C NMR (DMSO-d 6 , 151 MHz) $\delta 182.24,166.73,124.06,123.83,51.93,37.86,31.40$.

HRMS $m / z$ (FAB, [M-Br-CO-2H] $]^{+}$: calculated for $\mathrm{C}_{11} \mathrm{H}_{14} \mathrm{~N}_{4} \mathrm{Ir} 395.0848$, found: 395.0845 .

EA calculated for $\mathrm{C}_{12} \mathrm{H}_{16} \mathrm{BrIrN}_{4} \mathrm{O}$ (wt 504.01): C, 28.60; H, 3.20; N, 11.12, found: C, 30.24; H, 3.22; N $10.39 \%$.

IR (neat, $\left.\mathrm{cm}^{-1}\right)$ : 2056, $1985 \mathrm{~cm}^{-1}$.

Mp decomposed at $245{ }^{\circ} \mathrm{C}$. 


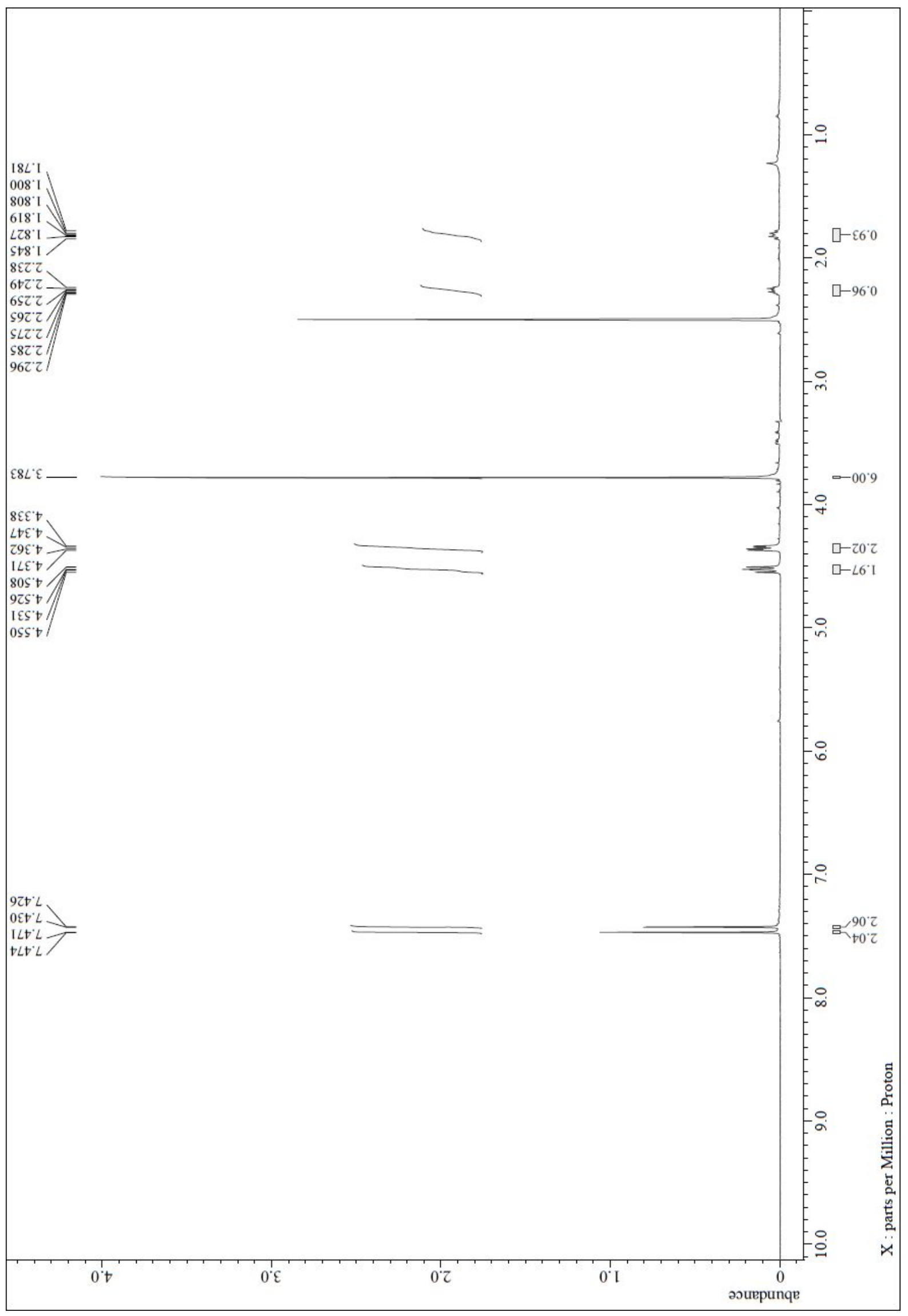




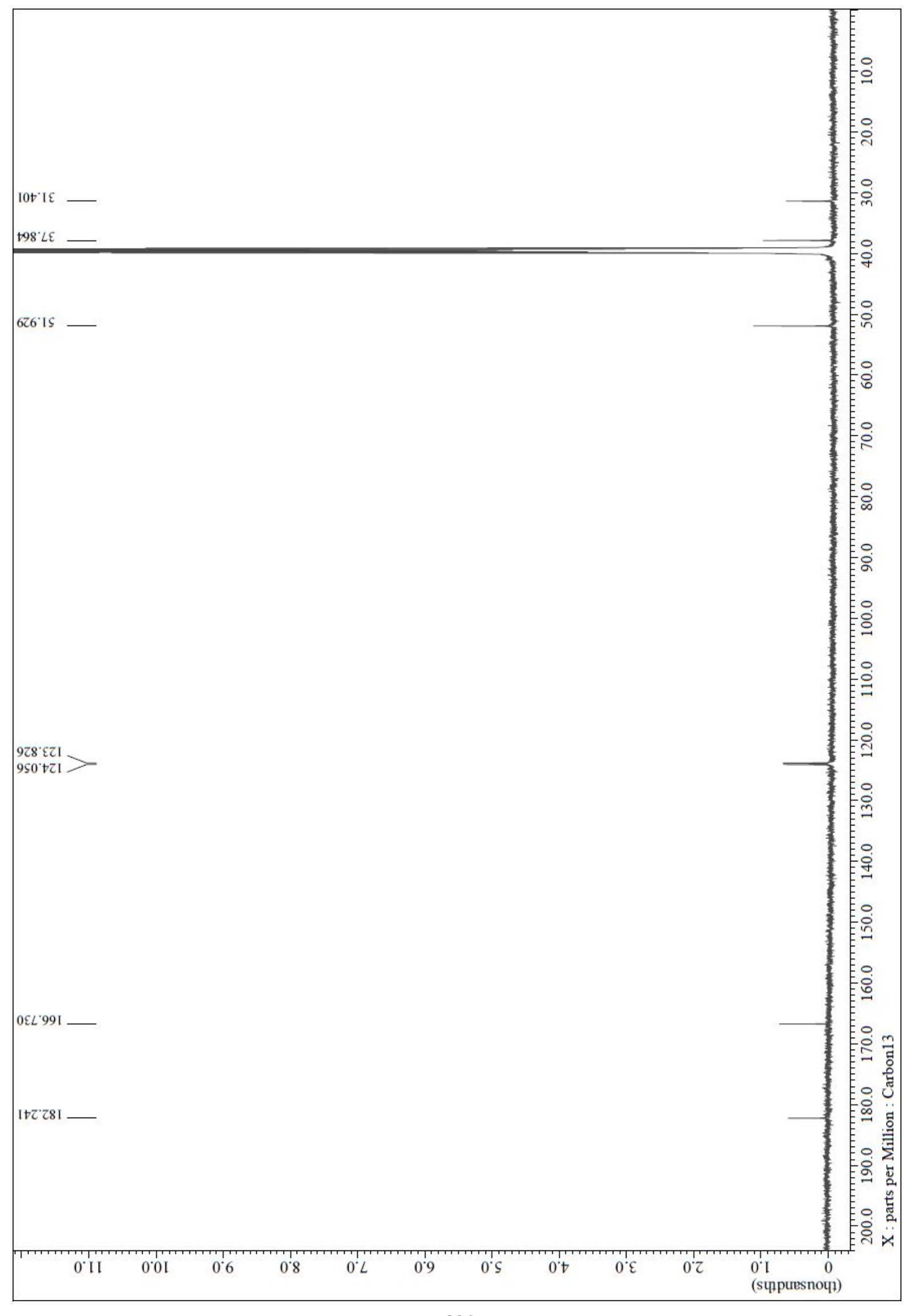




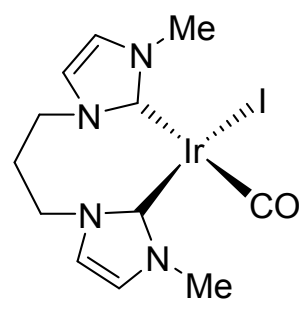

$\operatorname{Ir}\left(\right.$ LMe3)(CO)I ( $\left.2^{\prime}\right)$ :

${ }^{1}$ H NMR (DMSO-d $\left.6,600 \mathrm{MHz}\right) \delta 7.47$ (d, $\left.J=1.7 \mathrm{~Hz}, 2 \mathrm{H}\right), 7.43(\mathrm{~d}, J=1.4 \mathrm{~Hz}, 2 \mathrm{H}), 4.53(\mathrm{dd}, J=14.3,11.9 \mathrm{~Hz}$, $2 \mathrm{H}), 4.36$ (dd, $J=14.5,5.5 \mathrm{~Hz}, 2 \mathrm{H}), 3.78$ (s, 6H), 2.24-2.29 (m, 1H), 1.79-1.85 (m, 1H).

${ }^{13}$ C NMR (DMSO-d 6 , 151 MHz) $\delta 182.19,166.72,124.04,123.79,51.91,37.85,31.38$.

HRMS $m / z$ (FAB, [M-I-CO-2H] $]^{+}$: calculated for $\mathrm{C}_{11} \mathrm{H}_{14} \mathrm{~N}_{4} \mathrm{Ir}$ 395.0848, found: 395.0851 .

EA calculated for $\mathrm{C}_{12} \mathrm{H}_{16} \operatorname{IIrN}_{4} \mathrm{O}$ (wt 551.41): C, 26.14; H, 2.93; N, 10.16, found: C, 26.18; H, 2.80; N, $10.07 \%$.

IR (neat, $\left.\mathrm{cm}^{-1}\right)$ : 2057, $1981 \mathrm{~cm}^{-1}$.

Mp decomposed at $235{ }^{\circ} \mathrm{C}$. 


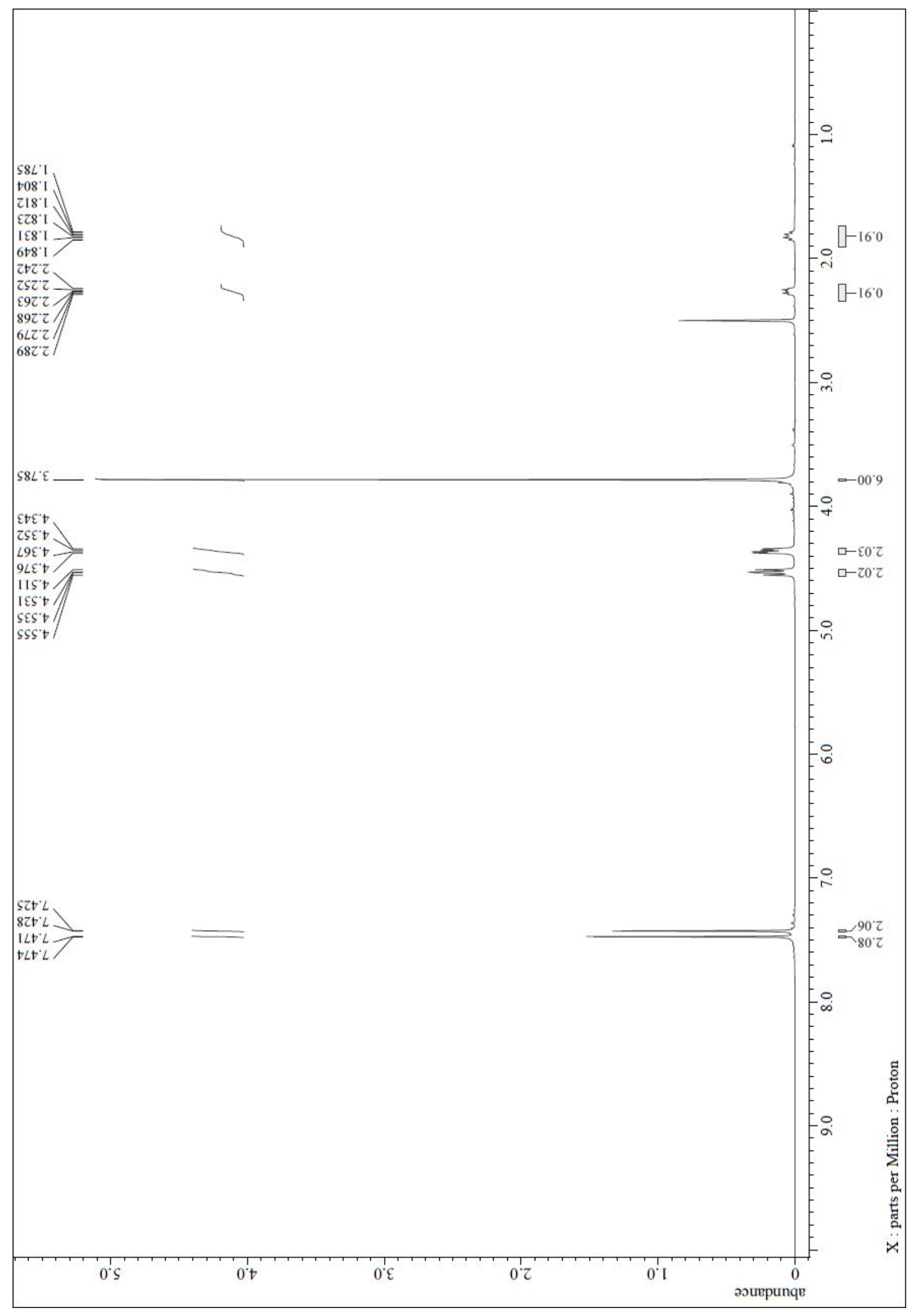




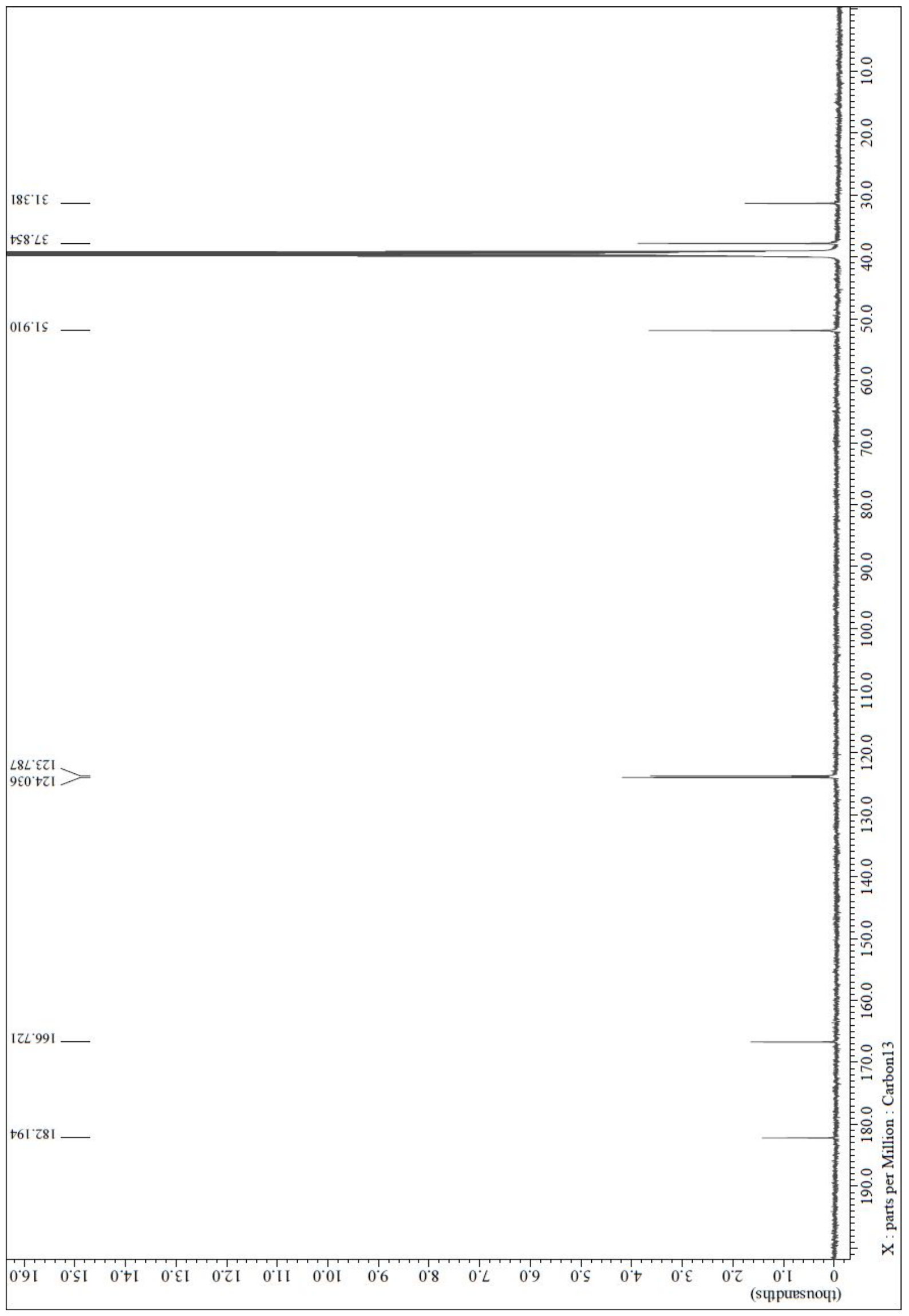




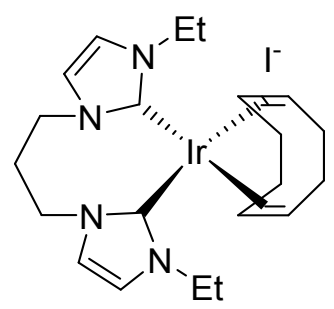

$\operatorname{Ir}(\operatorname{LEt} 3)\left(\right.$ COD)I ( $\left.1^{\prime \prime}\right)$ :

${ }^{1}$ H NMR (DMSO-d $\left.6,600 \mathrm{MHz}\right) \delta 7.32(\mathrm{~d}, J=2.1 \mathrm{~Hz}, 2 \mathrm{H}), 7.31(\mathrm{~d}, J=2.1 \mathrm{~Hz}, 2 \mathrm{H}), 4.58(\mathrm{dd}, J=14.1,10.7 \mathrm{~Hz}$, 2H), $4.40(\mathrm{td}, J=13.9,6.9 \mathrm{~Hz}, 2 \mathrm{H}), 4.30(\mathrm{dd}, J=14.4,6.6 \mathrm{~Hz}, 2 \mathrm{H}), 4.06(\mathrm{td}, J=13.9,6.9 \mathrm{~Hz}, 2 \mathrm{H}), 3.97-4.01(\mathrm{~m}$, $4 \mathrm{H}), 2.25-2.35(\mathrm{~m}, 5 \mathrm{H}), 1.88-10.99(\mathrm{~m}, 4 \mathrm{H}), 1.69-1.75(\mathrm{~m}, 1 \mathrm{H}), 1.34(\mathrm{t}, J=7.2 \mathrm{~Hz}, 6 \mathrm{H})$.

${ }^{13}$ C NMR (DMSO-d 6 , $151 \mathrm{MHz}$ ) $\delta 175.85,123.02,119.75,75.43,73.86,51.48,44.58,33.07,30.78,30.73,15.93$.

HRMS $m / z$ (FAB, [M-I] $]^{+}$calculated for $\mathrm{C}_{21} \mathrm{H}_{32} \mathrm{~N}_{4} \mathrm{Ir} 533.2257$, found: 533.2259 .

EA calculated for $\mathrm{C}_{21} \mathrm{H}_{32} \mathrm{IIrN}_{4}$ (wt 659.64): C, 38.24; H, 4.89; N, 8.49, found: C, 38.30; H, 4.87; N, $8.33 \%$. Mp $230{ }^{\circ} \mathrm{C}$. 


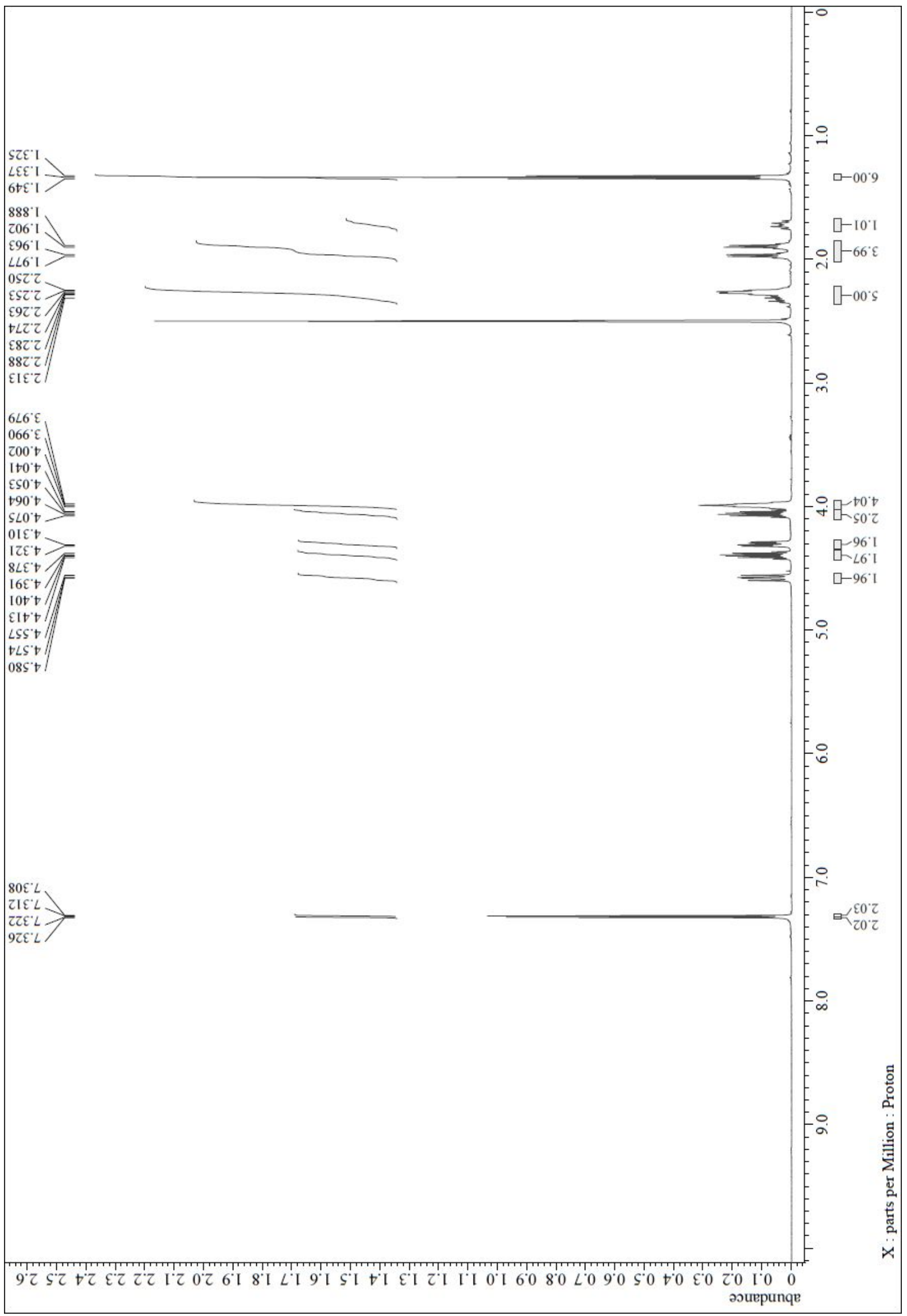




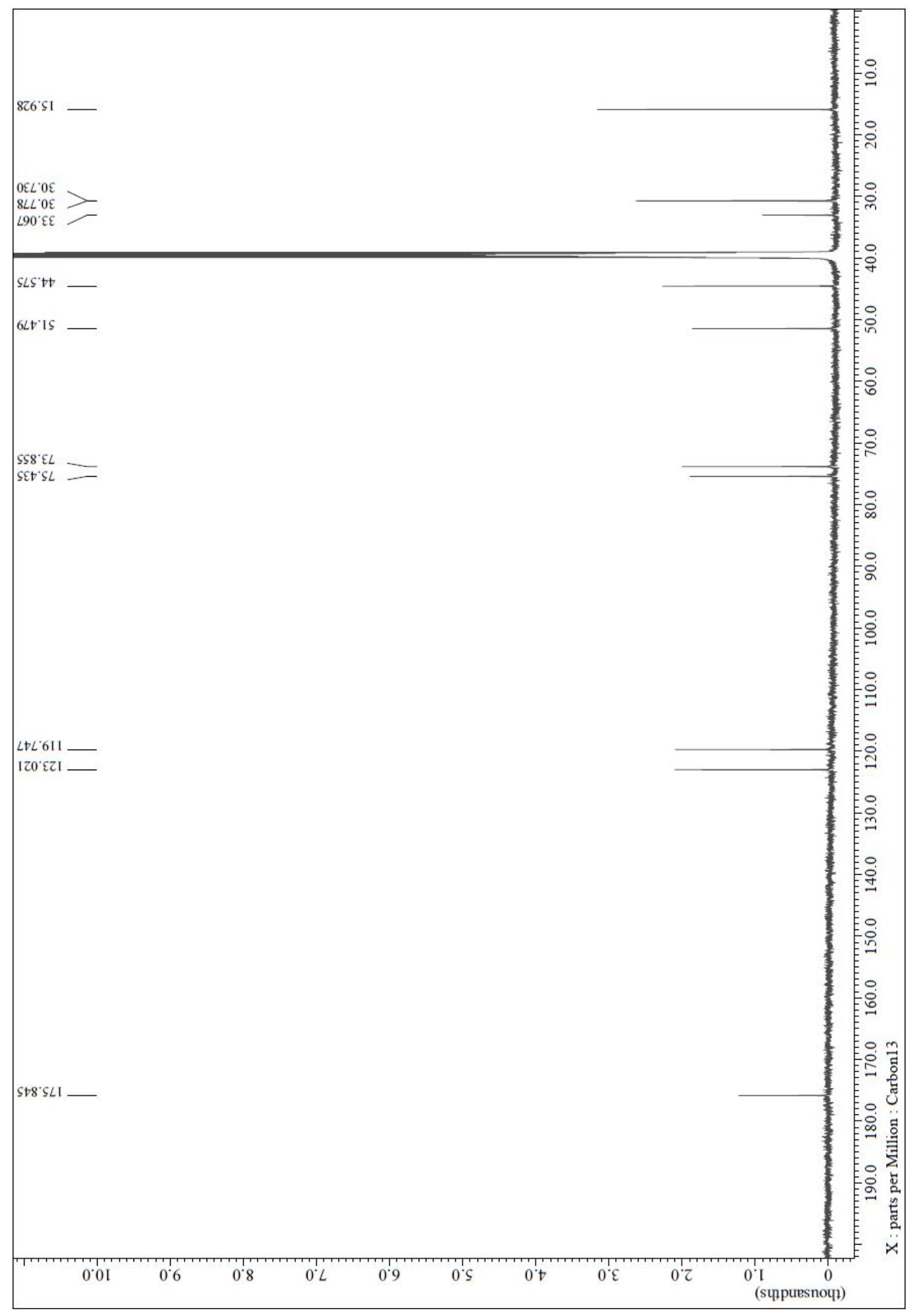




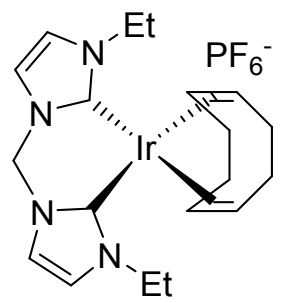

\section{$\operatorname{Ir}($ LEt1)(COD)I·KPF 6 THF (6):}

${ }^{1} \mathbf{H}$ NMR $\left(\mathrm{CD}_{2} \mathrm{Cl}_{2}-\mathrm{d}_{2}, 600 \mathrm{MHz}\right) \delta 7.69(\mathrm{~d}, J=2.1 \mathrm{~Hz}, 2 \mathrm{H}), 7.25(\mathrm{~s}, 2 \mathrm{H}), 6.91(\mathrm{~d}, J=2.1 \mathrm{~Hz}, 2 \mathrm{H}), 4.76(\mathrm{t}, J=2.8$ $\mathrm{Hz}, 4 \mathrm{H}), 4.37$ (dtd, $J=70.3,14.0,6.8 \mathrm{~Hz}, 4 \mathrm{H}), 2.93-3.04$ (m, 4H), 2.11-2.25 (m, 8H), 1.77-1.85 (m, 4H), 1.46 (t, $J=7.2 \mathrm{~Hz}, 6 \mathrm{H})$.

${ }^{13} \mathrm{C}$ NMR $\left(\mathrm{CD}_{2} \mathrm{Cl}_{2}-\mathrm{d}_{2}, 151 \mathrm{MHz}\right) \delta 180.18,121.30,121.09,84.02,61.53,56.40,55.99,45.76,33.56,32.97,30.72$, $30.59,15.76$.

HRMS $m / z\left(\mathrm{FAB},\left[\mathrm{M}-\mathrm{PF}_{6}\right]^{+}\right.$: calculated for $\mathrm{C}_{19} \mathrm{H}_{28} \mathrm{~N}_{4} \mathrm{Ir}$ 505.1944, found: 505.1942 .

EA calculated for $\mathrm{C}_{23} \mathrm{H}_{36} \mathrm{~F}_{6} \mathrm{IIKKN}{ }_{4} \mathrm{OP}$ (wt 887.752): C, 31.12; H, 4.09; N, 6.31, found: $\mathrm{C}, 30.93 ; \mathrm{H}, 3.80 ; \mathrm{N}, 5.28 \%$. Mp decomposed at $220{ }^{\circ} \mathrm{C}$. 


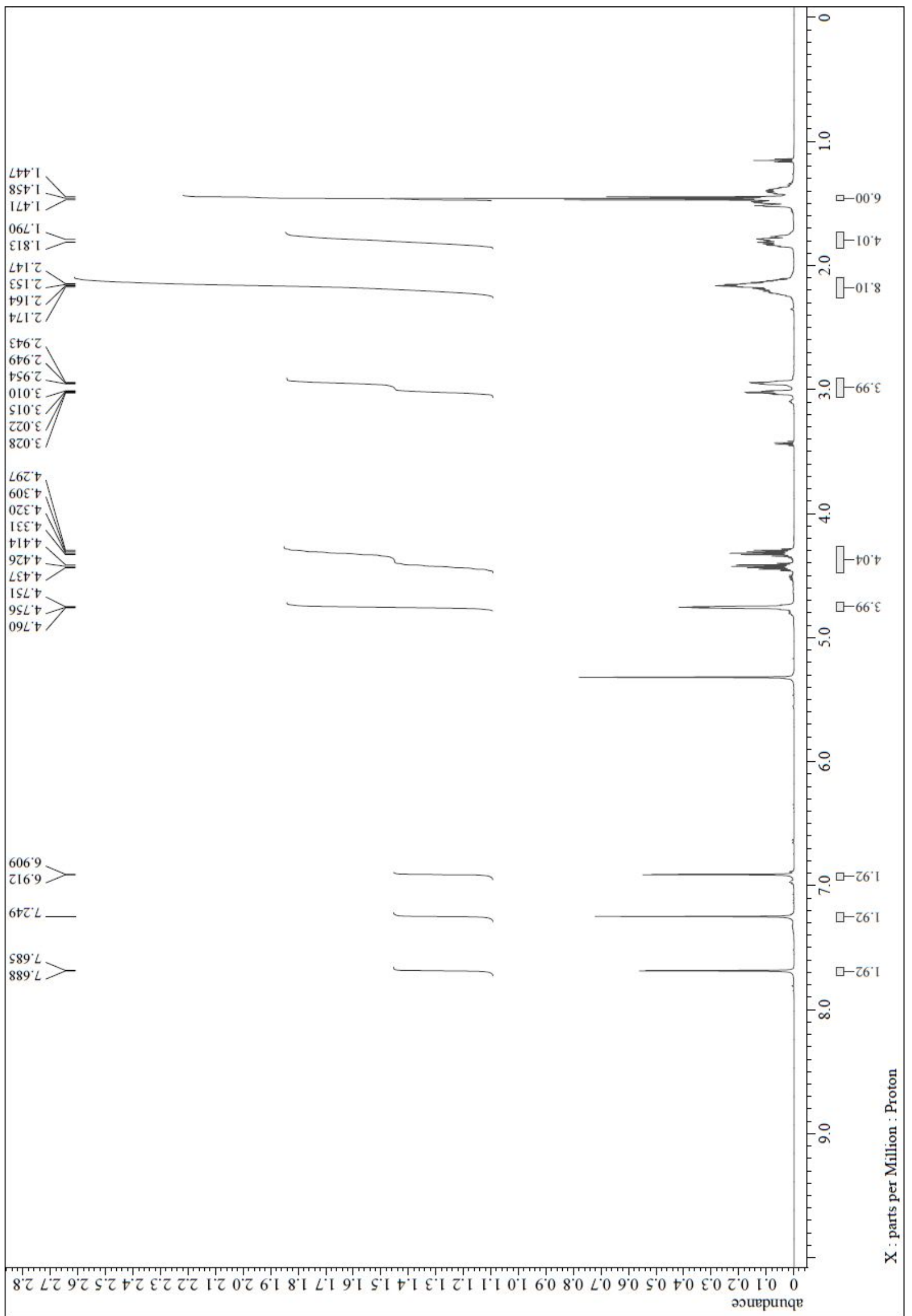




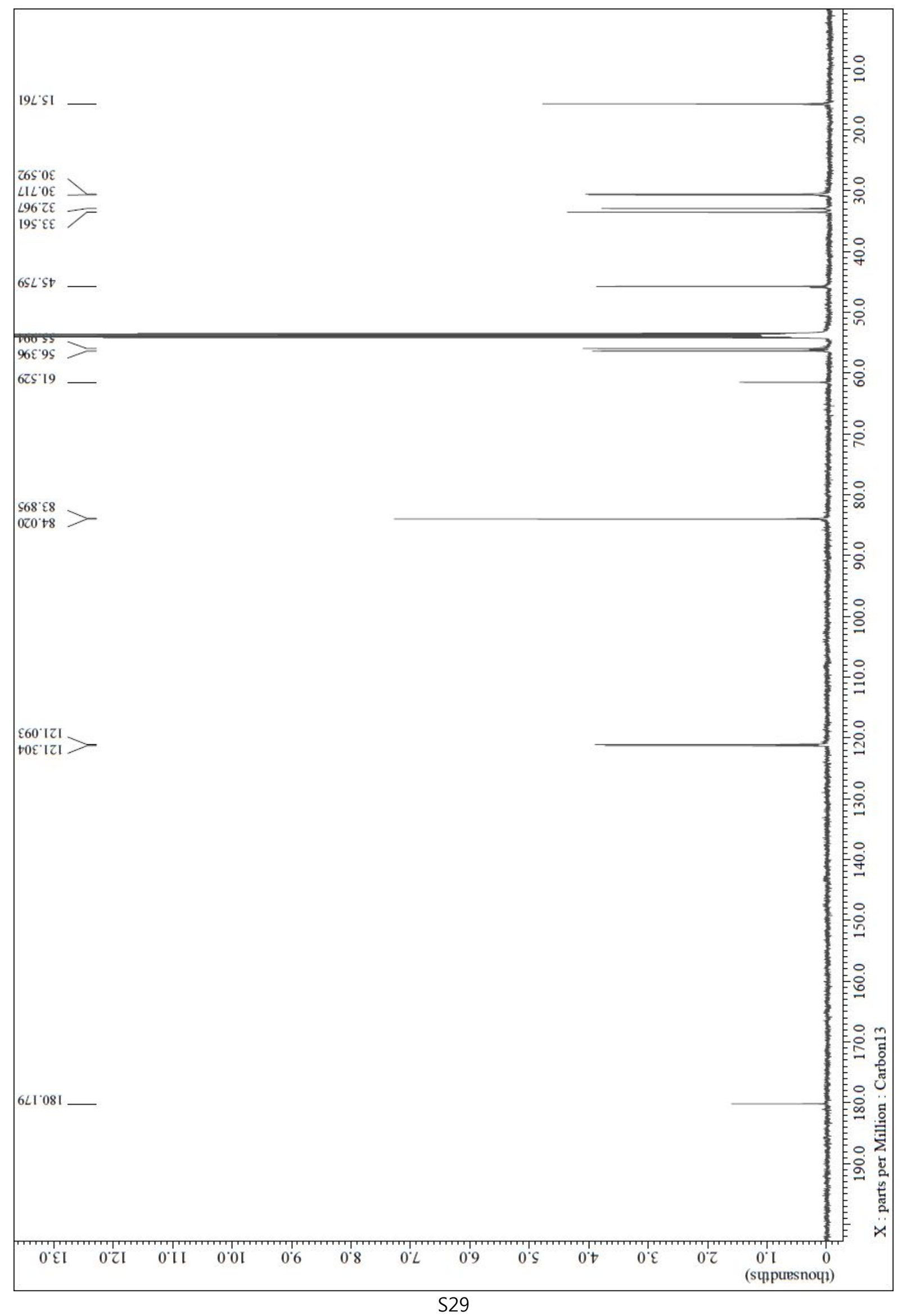




\section{Analysis of recovered Ir catalyst 1 from the reaction mixture}

${ }^{1} \mathrm{H}$ NMR of recovered Ir catalyst 1: Since the concentration of the catalyst was very low in the reaction, the characterization of recovered catalysts was not possible. The biscarbene protons (imidazolium rings) of the Ir complex were speculated in the ${ }^{1} \mathrm{H}$ NMR spectrum of the recovered mixture.
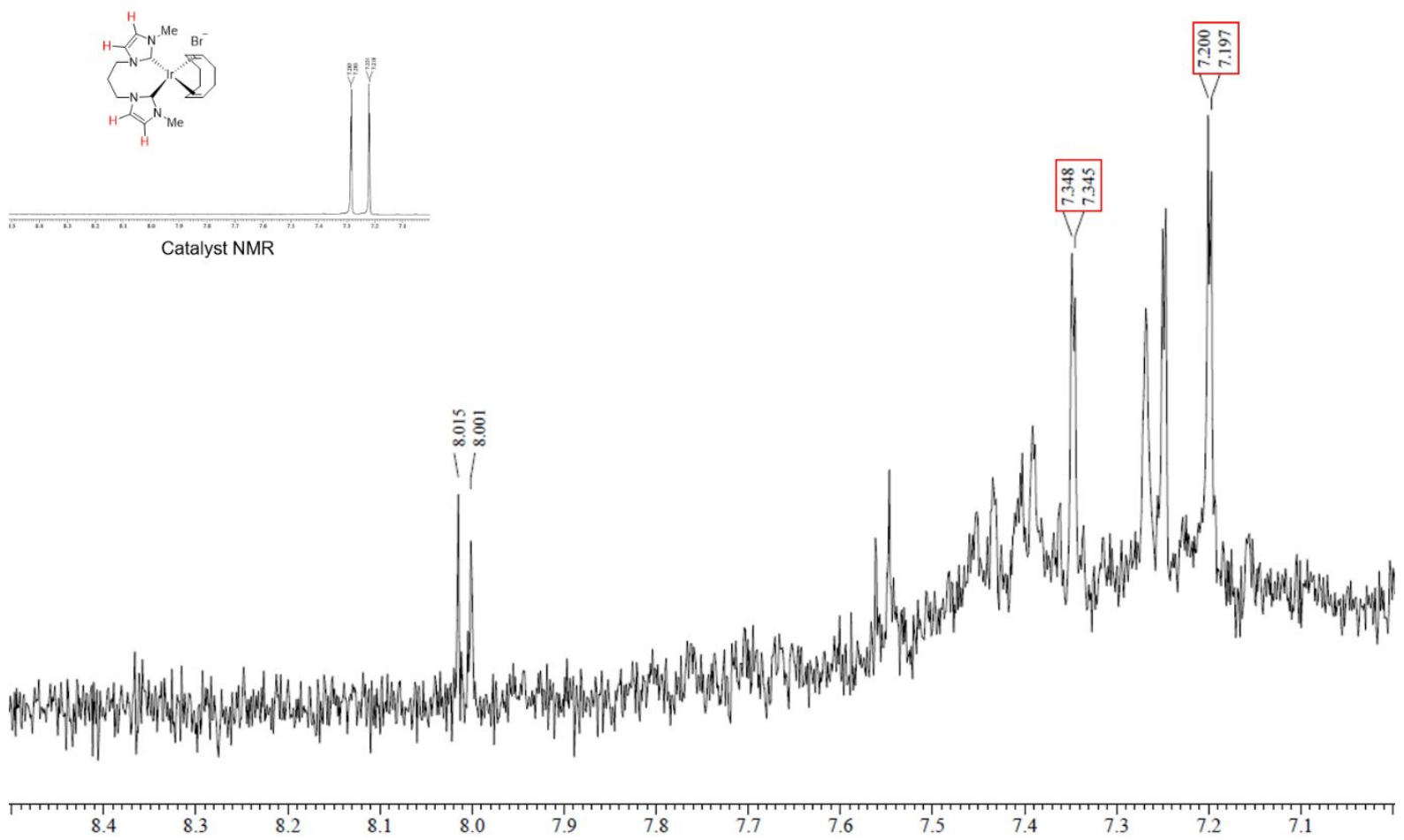


\section{DFT calculation results}

\section{Computation details}

A computational study based on density functional theory (DFT) was performed to understand the change of catalytic activity closely depending on the structure of $\operatorname{Ir}(\mathrm{I})$ catalyst, i.e., the tether group between two carbene ligands. The hybrid PBE0 functional ${ }^{3}$ combined with the Grimme's D3 method $^{4}$ was utilized to effectively describe the non-bonding interaction between reagents. The molecular geometries were optimized using 6$31 \mathrm{G}(\mathrm{d}, \mathrm{p})$ basis set, and then the single-point energy calculations were performed using $6-311++\mathrm{G}(\mathrm{d}, \mathrm{p})$ basis set to improve computational accuracy. The LanL2DZ ${ }^{5}$ and $\mathrm{SDD}^{6}$ were employed as an effective pseudo-potential for iridium for geometry optimization and single point calculation, respectively. The frequency calculations were carried out with the same functional and basis set as those used in the geometry optimization not only to confirm local minima but also to obtain thermal contribution to the reaction energy diagram which are corrected by Gibbs free energy obtained at $298.15 \mathrm{~K}$ and $1 \mathrm{~atm}$. The polarizable continuum model using the integral equation formalism (IEFPCM) was employed to take account for the influence of solvent medium (glycerol, dielectric constant $\varepsilon=42.5$ ) on geometric and electronic structures. ${ }^{7}$ For verifying the reliability of computational results, we also performed the DFT calculations using MN15 functional ${ }^{8}$ and obtained a consistent result with PBE0 calculations (Fig. S1). Natural population analysis was performed to elucidate the atomic charge of central Ir atom during catalytic reaction, which are obtained using electron density obtained by PBE0-D3/6-311++G(d,p)//PBE0D3/6-31G(d,p) calculations. All calculations were performed using Gaussian 16 program suite. ${ }^{9}$

\footnotetext{
${ }^{3}$ Adamo C. and Barone, V. Toward reliable density functional methods without adjustable parameters: the PBEO model. J. Chem. Phys. 1999, $110,6158-6170$.

${ }^{4}$ Grimme, S.; Ehrlich, S. and Goerigk, L. Effect of the damping function in dispersion corrected density functional theory. J. Comp. Chem. 2011, 32, 1456-1465.

${ }^{5}$ Hay P. J. and Wadt, W. R. Ab initio effective core potentials for molecular calculations. Potentials for the transition metal atoms Sc to Hg. J. Chem. Phys. 1985, 82, 299-310.

${ }^{6}$ Andrae, D.; Haeussermann, U.; Dolg, M.; Stoll, H. and Preuss, H. Energy-adjusted ab initio pseudopotentials for the second and third row transition elements. Theor. Chem. Acc. 1990, 77, 123-141.

${ }^{7}$ Tomasi, J.; Mennucci, B. and Cammi, R. Quantum mechanical continuum solvation models. Chem. Rev. 2005, 105, $2999-3094$.

${ }^{8}$ Yu, H. S.; He, X.; Li, S. L. and Truhlar, D. G. MN15: A Kohm-Sham global-hybrid exchange-correlation density functional with broad accuracy for multi-reference and single-reference systems and noncovalent interactions. Chem. Sci. 2016, 7, 5032-5051.

${ }^{9}$ Reed, A. E.; Weinstock, R. B. and Weinhold, F. Natural population analysis. J. Chem. Phys. 1985, 83, 735-746.

M. J. Frisch, G. W. Trucks, H. B. Schlegel, G. E. Scuseria, M. A. Robb, J. R. Cheeseman, G. Scalmani, V. Barone, G. A. Petersson, H. Nakatsuji, X. Li, M. Caricato, A. V. Marenich, J. Bloino, B. G. Janesko, R. Gomperts, B. Mennucci, H. P. Hratchian, J. V. Ortiz, A. F. Izmaylov, J. L. Sonnenberg, D. Williams-Young, F. Ding, F. Lipparini, F. Egidi, J. Goings, B. Peng, A. Petrone, T. Henderson, D. Ranasinghe, V. G. Zakrzewski, J. Gao, N. Rega, G. Zheng, W. Liang, M. Hada, M. Ehara, K. Toyota, R. Fukuda, J. Hasegawa, M. Ishida, T. Nakajima, Y. Honda, O. Kitao, H. Nakai, T. Vreven, K. Throssell, J. A. Montgomery, Jr., J. E. Peralta, F. Ogliaro, M. J. Bearpark, J. J. Heyd, E. N. Brothers, K. N. Kudin, V. N. Staroverov, T. A. Keith, R. Kobayashi, J. Normand, K. Raghavachari, A. P. Rendell, J. C. Burant, S. S. Iyengar, J. Tomasi, M. Cossi, J. M. Millam, M. Klene, C. Adamo, R. Cammi, J. W. Ochterski, R. L. Martin, K. Morokuma, O. Farkas, J. B. Foresman, D. J. Fox, Gaussian 16, Revision B.01, Gaussian, Inc., Wallingford CT, 2016.
} 


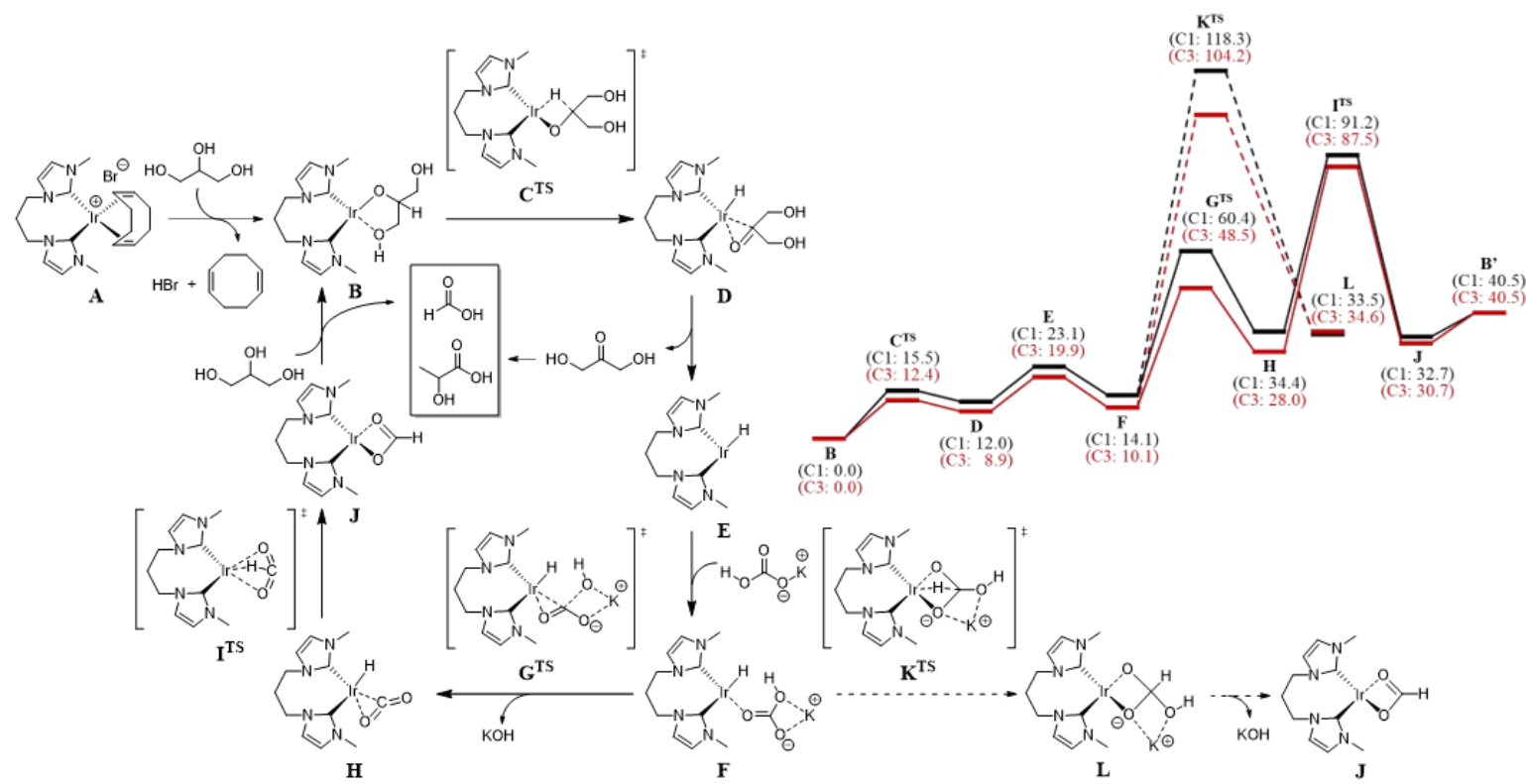

Figure S3. Proposed reaction mechanism for the simultaneous conversion of inorganic carbonate/glycerol to formate/lactate and the corresponding reaction energy diagram for two catalysts, $\mathbf{1}$ and its tether-modified catalyst, where we modified the length of the alkyl chain tether from $n=3$ (1) to $n=1$. C3 and C1 denote catalyst 1 and its tether-modified catalyst, respectively. The direct reduction process of bicarbonate bound on the iridium catalyst is indicated as $\mathbf{F} \rightarrow \mathbf{K}^{\mathbf{T S}} \rightarrow \mathbf{L}$, which should be followd by dihydroxylation $(\mathbf{L} \rightarrow \mathbf{J})$ to produce formates. Because the reaction barrier for dihydroxylation $(\mathbf{L} \rightarrow \mathbf{J})$ is expected to be lower than that for direct reduction of inorganic carbonate by hydride transfer $\left(\mathbf{F} \rightarrow \mathbf{K}^{\mathbf{T S}} \rightarrow \mathbf{L}\right)$ as observed in $\mathbf{F} \rightarrow \mathbf{G}^{\mathbf{T S}} \rightarrow \mathbf{H} \rightarrow \mathbf{I}^{\mathrm{TS}} \rightarrow \mathbf{J}$, the transition state between $\mathbf{L}$ and $\mathbf{J}$ was not elucidated. A reaction energy diagram was constructed using PBE0-D3/6-311++G(d,p)//PBE0D3/6-31G(d,p) calculations (see Figure S4 for the reaction energy diagram constructed using MN15/6$311++\mathrm{G}(\mathrm{d}, \mathrm{p}) / / \mathrm{MN} 15 / 6-31 \mathrm{G}(\mathrm{d}, \mathrm{p})$ calculations $)$. All the energy values are related to the Gibbs free energy $(\Delta \mathrm{G}$ in $\mathrm{kcal} / \mathrm{mol}$ ) at $298.15 \mathrm{~K}$ and $1 \mathrm{~atm}$. 


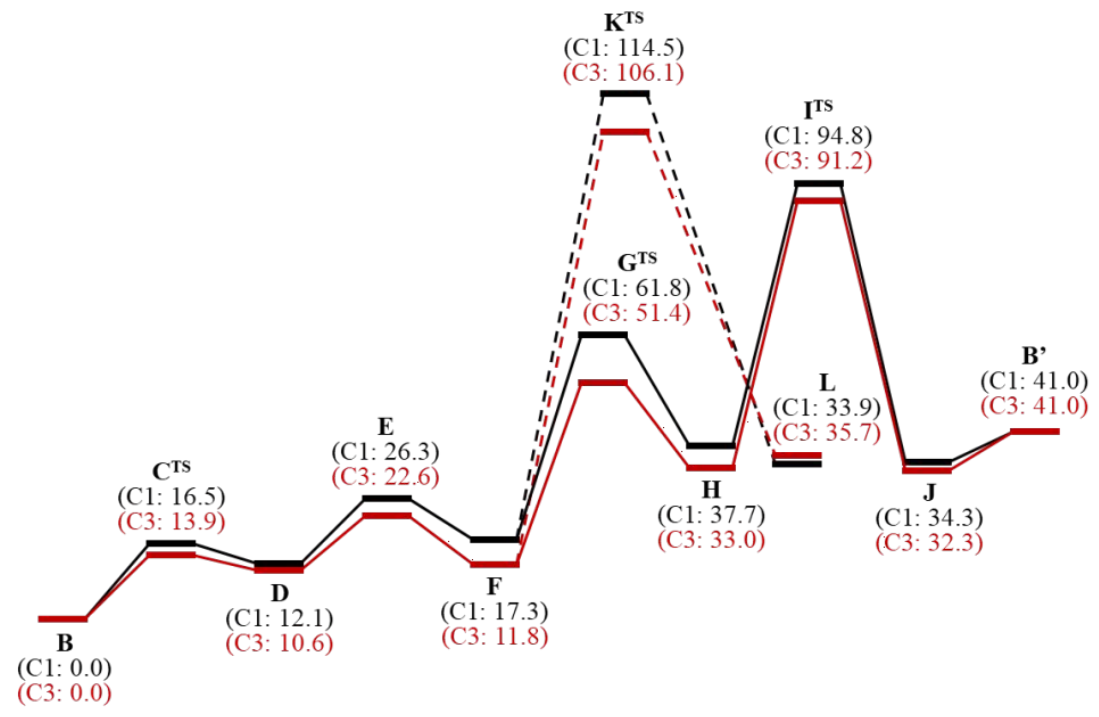

Figure S4. Reaction energy diagram for two catalysts, $\mathbf{1}$ and its tether-modified catalyst, where we modified the length of alkyl chain tether from $n=3$ (1) to $n=1$. The $\mathrm{C} 3$ and $\mathrm{C} 1$ denote catalyst $\mathbf{1}$ and its tether-modified catalyst. The reaction energy diagram was constructed using $M N 15 / 6-311++G(d, p) / / M N 15 / 6-31 G(d, p)$ calculations. All energy values are corrected with Gibbs free energy $(\Delta \mathrm{G}$ in $\mathrm{kcal} / \mathrm{mol})$ at $298.15 \mathrm{~K}$ and $1 \mathrm{~atm}$. 


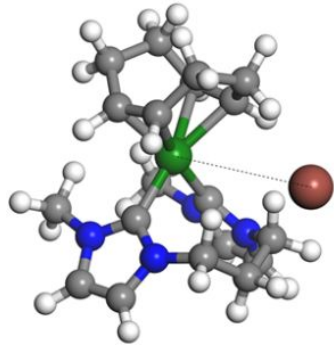

A

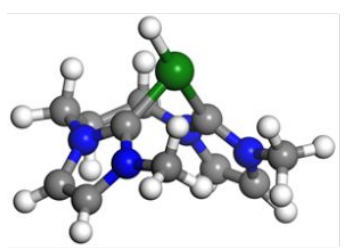

E

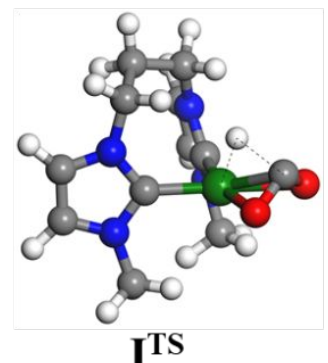

I $^{\mathrm{TS}}$

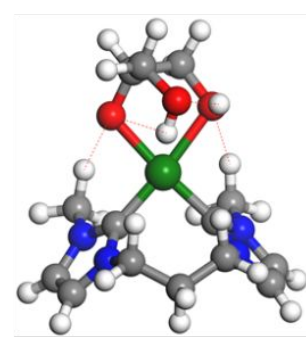

B

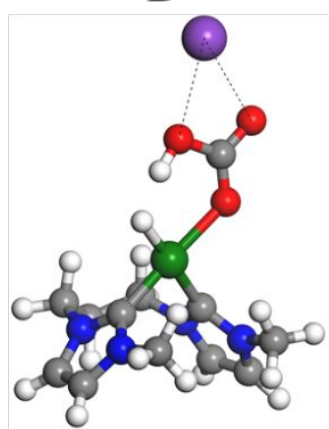

F

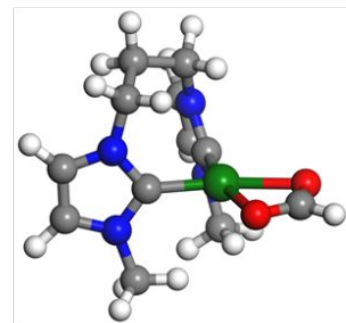

J

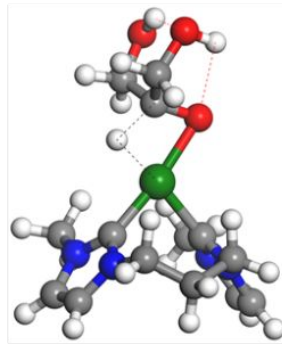

$\mathrm{C}^{\mathrm{TS}}$

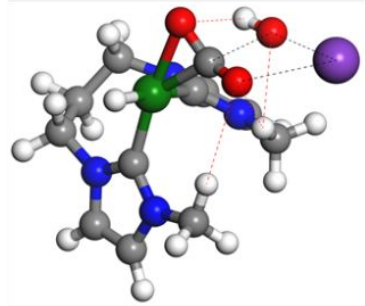

$G^{T S}$

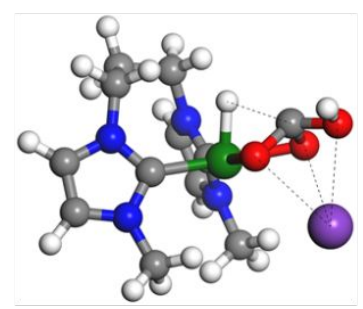

$\mathrm{K}^{\mathrm{TS}}$

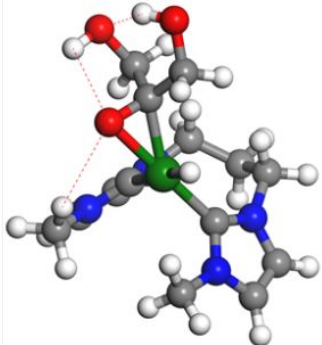

D

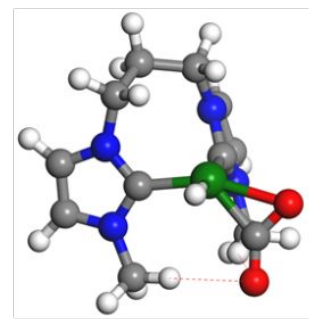

H

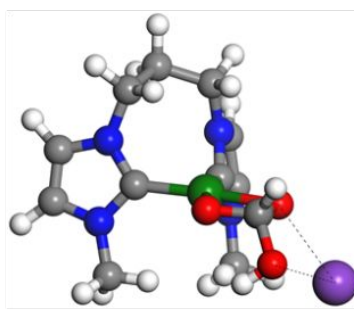

L

Figure S5. Optimized geometries of C3 (1) catalyst using PBE0-D3/6-31G(d,p) calculations along the proposed reaction mechanism for simultaneous conversion of inorganic carbonate/glycerol to formate/lactate (see Figure 3). The $\mathrm{O}-\mathrm{H} \ldots \mathrm{O}$ and $\mathrm{C}-\mathrm{H} \ldots \mathrm{O}$ hydrogen bonds are indicated with red-colored dashed lines. [Ir, green; Br, brown; $\mathrm{K}$, purple; $\mathrm{O}$, red; $\mathrm{N}$, blue; $\mathrm{C}$, gray; $\mathrm{H}$, white] 


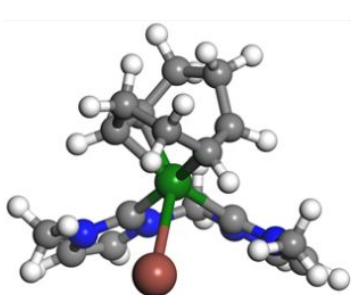

A

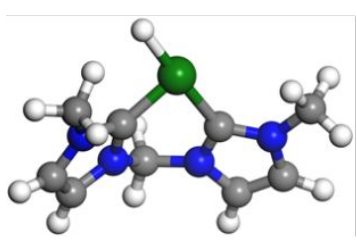

E

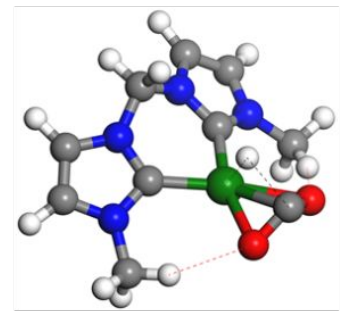

I $^{\mathrm{TS}}$

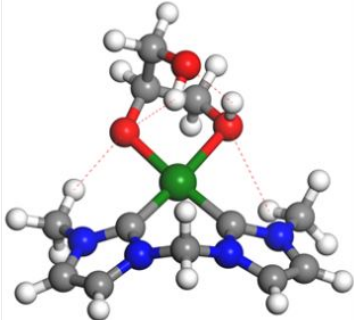

B

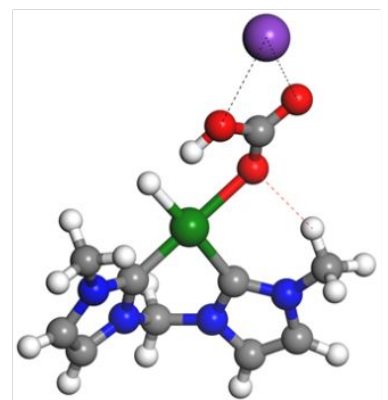

F

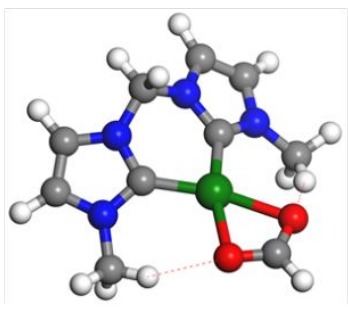

J

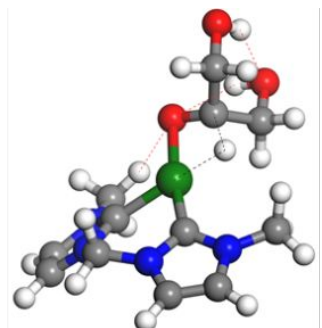

$\mathrm{C}^{\mathrm{TS}}$

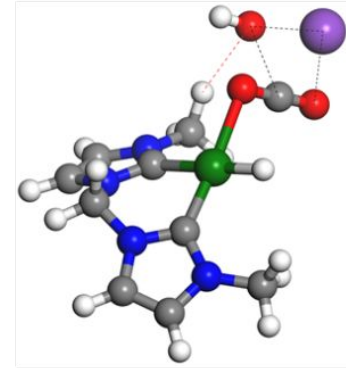

$\mathbf{G}^{\mathrm{TS}}$

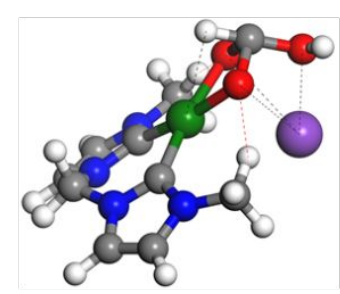

$\mathrm{K}^{\mathrm{TS}}$

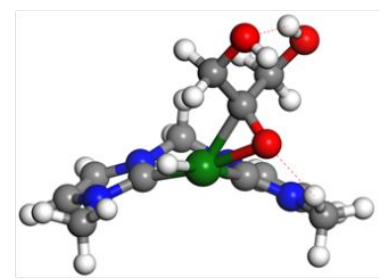

D

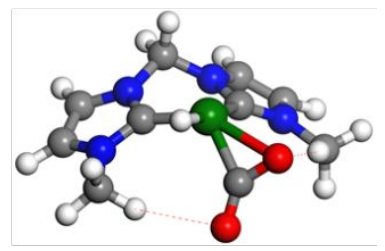

H

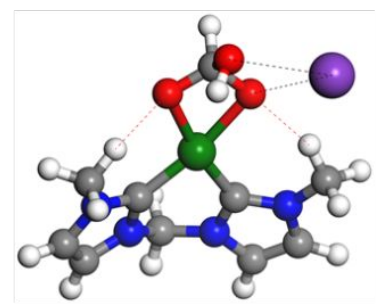

L

Figure S6. Optimized geometries of $\mathrm{C} 1$ catalyst using PBE0-D3/6-31G(d,p) calculations along the proposed reaction mechanism for simultaneous conversion of inorganic carbonate/glycerol to formate/lactate (see Figure 3). The O-H... O and C-H...O hydrogen bonds are indicated with red-colored dashed lines. [Ir, green; Br, brown; $\mathrm{K}$, purple; $\mathrm{O}$, red; $\mathrm{N}$, blue; $\mathrm{C}$, gray; $\mathrm{H}$, white] 
(a)

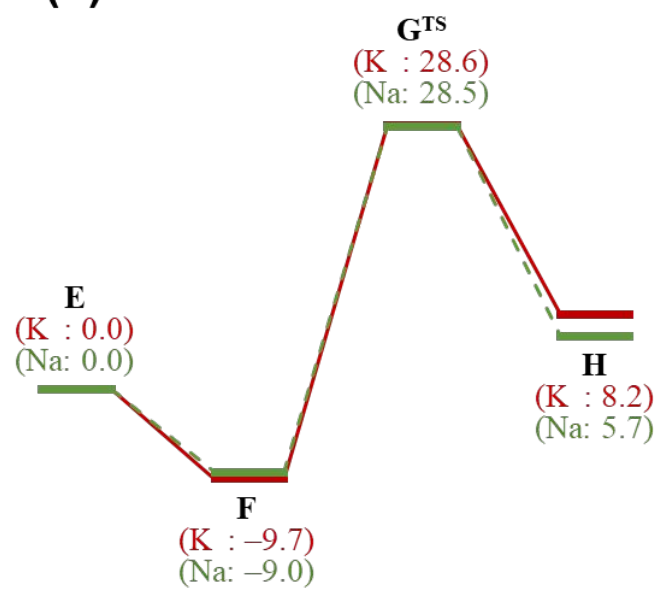

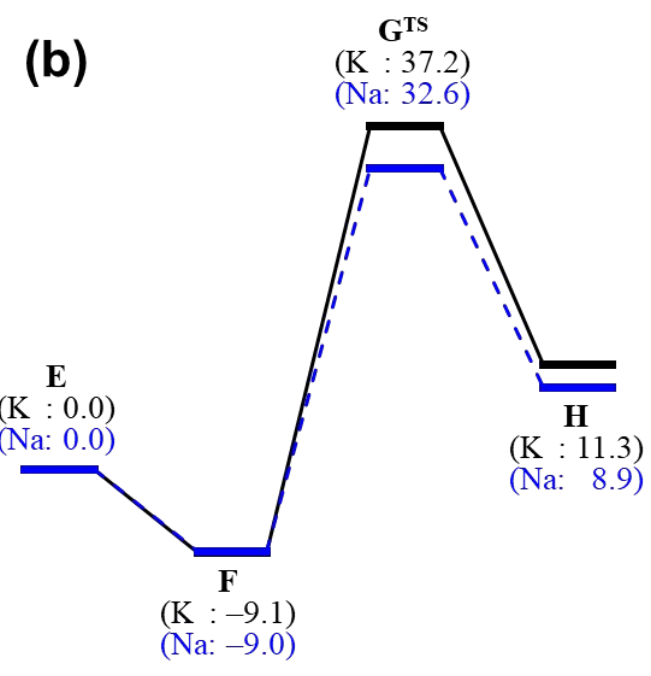

Figure S7. Reaction energy diagrams for $\mathbf{E} \rightarrow \mathbf{F} \rightarrow \mathbf{G}^{\mathbf{T S}} \rightarrow \mathbf{H}$ depending on the kind of inorganic bicarbonate, (a) $\mathrm{C} 3$ catalyst (red solid line: $\mathrm{KHCO}_{3}$, green dashed line: $\mathrm{NaHCO}_{3}$ ) and (b) $\mathrm{C} 1$ catalyst (black solid line: $\mathrm{KHCO}_{3}$, blue dashed line: $\left.\mathrm{NaHCO}_{3}\right)$. All reaction energy diagrams were constructed using PBE0-D3/6-311++G(d,p)//PBE0$\mathrm{D} 3 / 6-31 \mathrm{G}(\mathrm{d}, \mathrm{p})$ calculations, in which the relative energy values $(\Delta \mathrm{G}$ in $\mathrm{kcal} / \mathrm{mol}$ at $298.15 \mathrm{~K}$ and $1 \mathrm{~atm})$ are obtained with respect to the energy of step $\mathbf{E}$. 


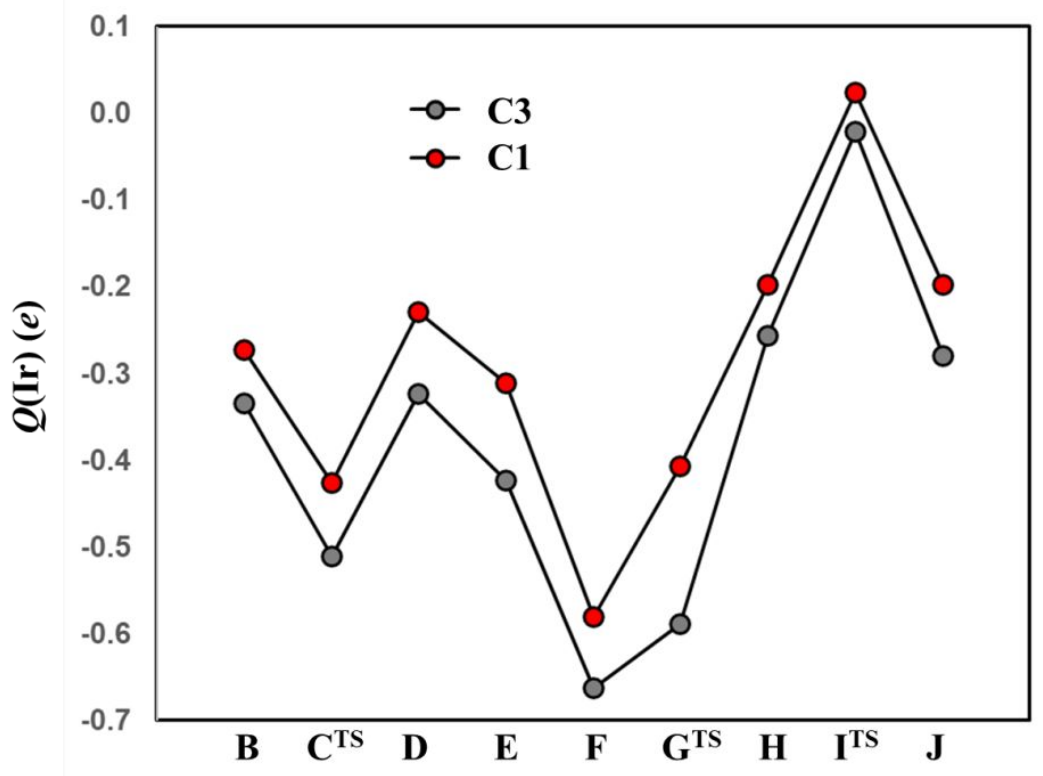

Figure S8. Atomic charge of central Ir atom during catalytic reaction. Natural population analysis was performed using electron density obtained from PBE0-D3/6-311++G(d,p)//PBE0-D3/6-31G(d,p) calculations. The LanL2DZ and SDD were employed as an effective pseudo-potential for iridium for geometry optimization and single point calculation, respectively. 

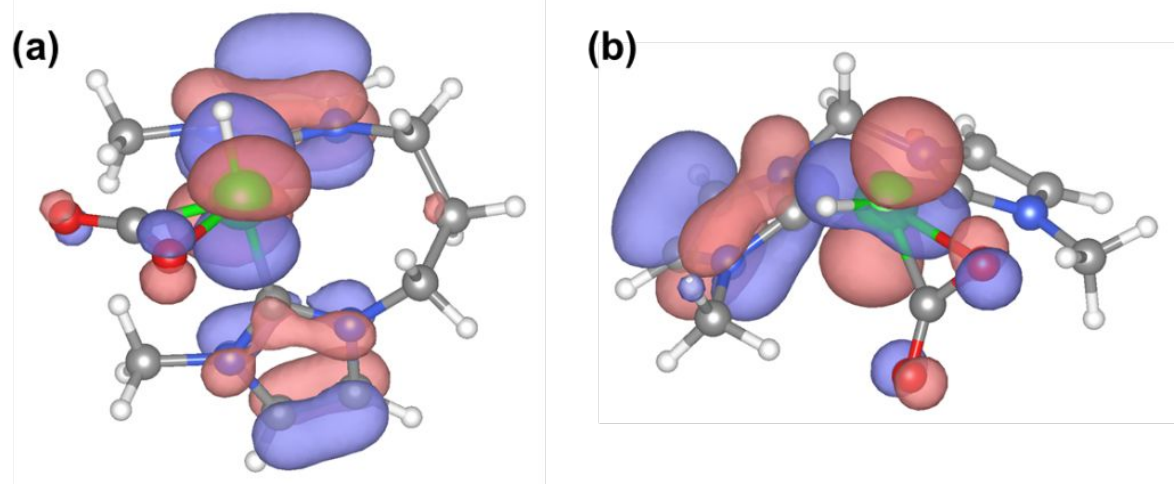

Figure S9. The highest occupied molecular orbitals (HOMO) of the $\mathrm{CO}_{2}$-coordiated $\mathrm{Ir}(\mathrm{I})-\mathrm{H}$ complex for (a) $\mathrm{C} 3$ and (b) $\mathrm{C} 1$ catalysts, which are obtained using PBE0-D3/6-311++G(d,p)//PBE0-D3/6-31G(d,p) calculations. The orbital axis for $\mathrm{O} \mathrm{p}_{\mathrm{z}}$ of $\mathrm{CO}_{2}$ in $\mathrm{C} 3$ catalyst is parallel to the orientation of $\mathrm{Ir} \mathrm{d}$ orbital, which indicates that $\mathrm{C} 3$ catalyst is capable to form more efficient $\pi$-interaction with $\mathrm{CO}_{2}$ compared to $\mathrm{C} 1$ catalyst. [iso-value $=0.03 \mathrm{e} / \mathrm{bohr}{ }^{3}$ ] 